\title{
3D AMR hydrosimulations of a compact source scenario for the Galactic Centre cloud G2
}

\author{
A. Ballone ${ }^{1,2}$, M. Schartmann ${ }^{3}$, A. Burkert ${ }^{1,2}$, S. Gillessen ${ }^{2}$, P. M. Plewa ${ }^{2}$, \\ R. Genzel ${ }^{2}$, O. Pfuhl ${ }^{2}$, F. Eisenhauer ${ }^{2}$, M. Habibi ${ }^{2}$, T. Ott ${ }^{2}$ and E. M. George ${ }^{2}$ \\ ${ }^{1}$ University Observatory Munich, Scheinerstraße 1, D-81679 München, Germany \\ ${ }^{2}$ Max-Planck-Institute for Extraterrestrial Physics, Postfach 1312, Giessenbachstraße, D-85741 Garching, Germany \\ ${ }^{3}$ Centre for Astrophysics and Supercomputing, Swinburne University of Technology, Hawthorn, Victoria 3122, Australia
}

Accepted XXX. Received YYY; in original form ZZZ

\begin{abstract}
The nature of the gaseous and dusty cloud G2 in the Galactic Centre is still under debate. We present three-dimensional hydrodynamical adaptive mesh refinement (AMR) simulations of G2, modeled as an outflow from a "compact source" moving on the observed orbit. The construction of mock position-velocity (PV) diagrams enables a direct comparison with observations and allow us to conclude that the observational properties of the gaseous component of G2 could be matched by a massive $\left(\dot{M}_{\mathrm{w}}=5 \times 10^{-7} M_{\odot} \mathrm{yr}^{-1}\right)$ and slow $\left(50 \mathrm{~km} \mathrm{~s}^{-1}\right)$ outflow, as observed for $\mathrm{T}$ Tauri stars. In order for this to be true, only the material at larger ( $>100 \mathrm{AU})$ distances from the source must be actually emitting, otherwise G2 would appear too compact compared to the observed PV diagrams. On the other hand, the presence of a central dusty source might be able to explain the compactness of G2's dust component. In the present scenario, 5-10 years after pericentre the compact source should decouple from the previously ejected material, due to the hydrodynamic interaction of the latter with the surrounding hot and dense atmosphere. In this case, a new outflow should form, ahead of the previous one, which would be the smoking gun evidence for an outflow scenario.
\end{abstract}

Key words: accretion, accretion disks - black hole physics - Galaxy: centre - ISM: clouds - stars: winds, outflows

\section{INTRODUCTION}

Since the date of its discovery, the nature of the little gaseous and dusty cloud G2 discovered by Gillessen et al. (2012) has remained enigmatic. The $\mathrm{Br} \gamma, \mathrm{Pa} \alpha$ and HeI recombination lines detected with the integral field spectrographs SINFONI $^{1}$ at the VLT (Gillessen et al. 2012, 2013a,b; Pfuhl et al. 2015; Valencia-S. et al. 2015) and OSIRIS $^{2}$ at the Keck telescope (Phifer et al. 2013) show a gas component extending both in size and velocity and following a high eccentricity Keplerian orbit (see the position-velocity diagrams in Gillessen et al. 2013a,b; Pfuhl et al. 2015). Gillessen et al. (2013b) and Pfuhl et al. (2015) have also reported the detection of a blue-shifted component, simultaneous with the red-shifted one, consistent with G2 passing pericentre as an extended object in March-April 2014. The detections in L' and $\mathrm{M}$ ' bands in the NIR with $\mathrm{NACO}^{3}$ at the VLT (Gillessen

\footnotetext{
1 http://www.eso.org/sci/facilities/paranal/instruments/sinfoni/

2 http://www2.keck.hawaii.edu/inst/osiris/

3 http://www.eso.org/sci/facilities/paranal/instruments/naco/
}

et al. 2012, 2013a) and with $\mathrm{NIRC}^{4}$ at the Keck telescope (Phifer et al. 2013; Witzel et al. 2014) suggest that G2 has an unresolved dust component at a temperature of roughly $550 \mathrm{~K}^{5}$

Several observational programs are currently monitoring its evolution ${ }^{6}$, also focusing on the interaction of this object with the extreme gravitational field of the $4.31 \times 10^{6} M_{\odot}$ supermassive black hole (SMBH) centred on SgrA* (Ghez et al. 2008; Gillessen et al. 2009) and with the hot and dense plasma accreting onto it. For example, increased emission in

${ }^{4}$ http://www2.keck.hawaii.edu/inst/nirc2/

5 The unresolved nature of the dust component shows that the latter is more compact than the gaseous one. As a matter of fact, the large point spread function (PSF) of NACO and NIRC2 makes the size of the dust emission only marginally in discrepancy with the sizes inferred from the $\operatorname{Br} \gamma$ emission. As shown by Witzel et al. (2014), the PSF in L' is also bigger than the tidal radius of a $2 M_{\odot}$ star, hence any dusty material might still be considerably extended and unbound from a possible central object.

${ }^{6}$ https://wiki.mpe.mpg.de/gascloud/FrontPage 
X-ray (Gillessen et al. 2012) and radio (Narayan et al. 2012; Sądowski et al. 2013b,a; Crumley \& Kumar 2013; Abarca et al. 2014) have been predicted by some models of the interaction of G2 with the outer accretion flow, but no consistent back reaction from either the accretion flow or SgrA* have been detected so far (Haggard et al. 2014; Chandler \& Sjouwerman 2014; Bower et al. 2015; Borkar et al. 2016). G2's partial or total disruption might also affect the accretion rate onto $\mathrm{SgrA}^{*}$ or affect the statistics and properties of flares from SgrA*: Ponti et al. (2015) showed that there has been an increase in the rate of X-ray bright flares since summer 2014, that might have been induced by G2's pericentre passage. However, this result is still under debate (Mossoux et al. 2016) and further monitoring of $\mathrm{SgrA}^{*}$ is needed to draw any strong conclusion. Finally, Plewa et al. (2017) have recently presented SINFONI and NACO observations of G2 in 2015 and 2016. In these, G2 appears to have passed pericentre, keeping on following more or less the same predicted orbit.

These observations are performed with the most up-todate instruments, pushing them to the limits of their capabilities; nonetheless, given the very small scales, it is still hard to evaluate the importance of the different physical processes in play. Trying to theoretically model the origin and fate of G2 has hence turned out to be challenging, but in the last two years several studies have shed light on this peculiar object.

The theoretical picture is presenting a dichotomy: G2 could be either a clump of diffuse gas and dust plunging into SgrA* or the outflow from a central source (possibly a young star) on a high eccentricity orbit around the SMBH.

The first scenario has been originally proposed by Gillessen et al. (2012). In this context, the gas is at a temperature $T \approx 10^{4} \mathrm{~K}$ and it is fully ionized by the ultraviolet (UV) radiation field produced by the nearby young and massive stars. Under the assumption of case$\mathrm{B}$ recombination and of a homogeneous sphere of radius $R_{c} \approx 1.9 \times 10^{15} \mathrm{~cm}$, these authors derived a mass of $M_{\mathrm{G} 2} \approx 1.7 \times 10^{28} \mathrm{~g} \approx 3$ Earth masses and a uniform density of $\rho_{c} \approx 6.1 \times 10^{-19} \mathrm{~g} \mathrm{~cm}^{-3}$. Several studies have been carried out for this scenario, focusing on the evolution of G2 and on its interaction with $\operatorname{Sgr}{ }^{*}$ 's accretion flow (Burkert et al. 2012; Schartmann et al. 2012; Anninos et al. 2012; Shcherbakov 2014; Schartmann et al. 2015). G2 seems to be followed by a larger component (named G2t or "the tail") following G2 on a similar orbit (Gillessen et al. 2013a; Pfuhl et al. 2015; Plewa et al. 2017) and Pfuhl et al. (2015) have shown that G2 could be connected to the previously discovered gas/dust cloud G1 (Clénet et al. 2004a,b, 2005; Ghez et al. 2005), whose orbit can be matched by a G2-like orbit after a drag force is applied to it (Pfuhl et al. 2015; McCourt \& Madigan 2016; Madigan et al. 2017; but see Plewa et al. 2017 for a different finding). This observational finding suggests that G2 is actually part of a much larger streamer. The idea of a gas streamer has been already proposed by Guillochon et al. (2014b), where the streamer could be produced by tidal stripping of the outer envelope of a late-type giant star, in a close encounter of such a star with the central SMBH. Another possibility for the origin of G2, if G2 is not linked to a central object, is clump formation through the non-linear thin shell instability in colliding winds of the outer O/WR stars (Calderón et al. 2016).
The second scenario involves a connection with a central source on G2's orbit. G2's Br $\gamma$ emission could either result from the gas lost by a photoevaporating disk (Murray-Clay \& Loeb 2012; Miralda-Escudé 2012) or by a photoevaporating starless (proto-)planet, tidally captured by the SMBH (Mapelli \& Ripamonti 2015; Trani et al. 2016) or produced by the interaction between an outflow from a low-mass star and the hot accretion flow (Scoville \& Burkert 2013; Ballone et al. 2013; De Colle et al. 2014; Zajaček et al. 2014, 2016, 2017) or a nova outburst (Meyer \& Meyer-Hofmeister 2012). Valencia-S. et al. (2015) tried to fully explain the $\mathrm{Br} \gamma$ line-width with a combination of an accretion stream and a disk wind close to a low-mass star. However, this is not in agreement with the PV diagrams obtained by Gillessen et al. (2013b) and Pfuhl et al. (2015), showing a spatially resolved velocity gradient consistent with tidal stretching. Finally, given the unresolved and constant-luminosity L'-band emission, Witzel et al. (2014) hypothesized that G2 is a binary star merger (see also Prodan et al. 2015; Stephan et al. 2016), forming a new low-mass $\left(<2 M_{\odot}\right)$ star and heating the dust component from inside. In a recent work, Ballone et al. (2016) showed that a relatively fast and massive outflow might also be able to reproduce both G2 and G2t at the same time, however neglecting the possible connection with the cloud G1. Differently from the present more quantitative study, focusing on reproducing only G2, the one in Ballone et al. (2016) is rather meant to be a proof of concept. As already mentioned, observations keep on hinting that G2 and G2t are closely related, but their connection is not fully established, yet. This led us to test both scenarios; the link and differences between the two studies are discussed in section 4.3 .

In this paper we focus on G2 only and present 3D simulations of an outflow scenario. Compared to the $2 \mathrm{D}$ simulations in Ballone et al. (2013), 3D simulations represent the geometry of the problem in a more realistic way and allow a much stricter comparison with the observations. Unfortunately, the high resolution used with the $2 \mathrm{D}$ simulations in Ballone et al. (2013) cannot be reached in this 3D study. So, the current simulations should be thought as complementary to the 2D ones presented in Ballone et al. (2013), rather than simple upgrades of them.

In Section 2 we describe the setup of our simulations. The results are presented in Section 3, where we compare them to the observations and we study the effect of the outflow parameters. Section 4 is dedicated to a more careful discussion of the ionization of the gas and the related uncertainties and of the numerical limitations. We also compare our study with previous ones and present the advantages and disadvantages of such a scenario. Summary and final remarks can be found in Section 5 .

\section{SIMULATION SETUP}

The simulations presented in this paper were run with the Eulerian code PLUTO (Mignone et al. 2007, 2012). Performing these three dimensional simulations with a uniform grid is computationally not feasible (see discussion in Ballone et al. 2013), so we adopted the adaptive mesh refinement (AMR) strategy, implemented in the code through the 
Table 1. Parameters of the simulated 3D AMR models.

\begin{tabular}{|c|c|c|c|c|c|}
\hline & $\dot{M}_{\mathrm{w}}\left(\mathrm{M}_{\odot} \mathrm{yr}^{-1}\right)$ & $v_{\mathrm{w}}(\mathrm{km} / \mathrm{s})$ & max resolution & coordinates & domain size $(x \times y \times z / R \times z)\left(10^{16} \mathrm{~cm}\right)$ \\
\hline standard model & $5 \times 10^{-7}$ & 50 & $\begin{array}{l}1.25 \times 10^{14} \mathrm{~cm} \\
8.3 \mathrm{AU}\end{array}$ & $\begin{array}{l}\text { 3D cartesian } \\
\text { (AMR) }\end{array}$ & {$[-26.4: 1.2] \times[-3.6: 4.8] \times[-2.4: 2.4]$} \\
\hline HV3D & $5 \times 10^{-7}$ & 250 & $\begin{array}{l}1.25 \times 10^{14} \mathrm{~cm} \\
8.3 \mathrm{AU}\end{array}$ & $\begin{array}{l}\text { 3D cartesian } \\
(\mathrm{AMR})\end{array}$ & {$[-28.8: 2.4] \times[-3.6: 7.2] \times[-4.8: 4.8]$} \\
\hline LMDOT3D & $10^{-7}$ & 50 & $\begin{array}{l}1.25 \times 10^{14} \mathrm{~cm} \\
8.3 \mathrm{AU}\end{array}$ & $\begin{array}{l}\text { 3D cartesian } \\
(\mathrm{AMR})\end{array}$ & {$[-26.4: 1.2] \times[-3.6: 4.8] \times[-2.4: 2.4]$} \\
\hline HMDOT3D & $2.5 \times 10^{-6}$ & 50 & $\begin{array}{l}1.25 \times 10^{14} \mathrm{~cm} \\
8.3 \mathrm{AU}\end{array}$ & $\begin{array}{l}\text { 3D cartesian } \\
(\mathrm{AMR})\end{array}$ & {$[-26.4: 1.2] \times[-3.6: 4.8] \times[-2.4: 2.4]$} \\
\hline stLOWRES & $5 \times 10^{-7}$ & 50 & $\begin{array}{l}2.5 \times 10^{14} \mathrm{~cm} \\
16.6 \mathrm{AU}\end{array}$ & $\begin{array}{l}\text { 3D cartesian } \\
(\mathrm{AMR})\end{array}$ & {$[-26.4: 1.2] \times[-3.6: 4.8] \times[-2.4: 2.4]$} \\
\hline st2D & $5 \times 10^{-7}$ & 50 & $\begin{array}{l}1.25 \times 10^{14} \mathrm{~cm} \\
8.8 \mathrm{AU}\end{array}$ & $\begin{array}{l}\text { 2D cylindrical } \\
\text { (fixed grid) }\end{array}$ & {$[0.0: 1.8] \times[-28.8:-3.0]$} \\
\hline
\end{tabular}
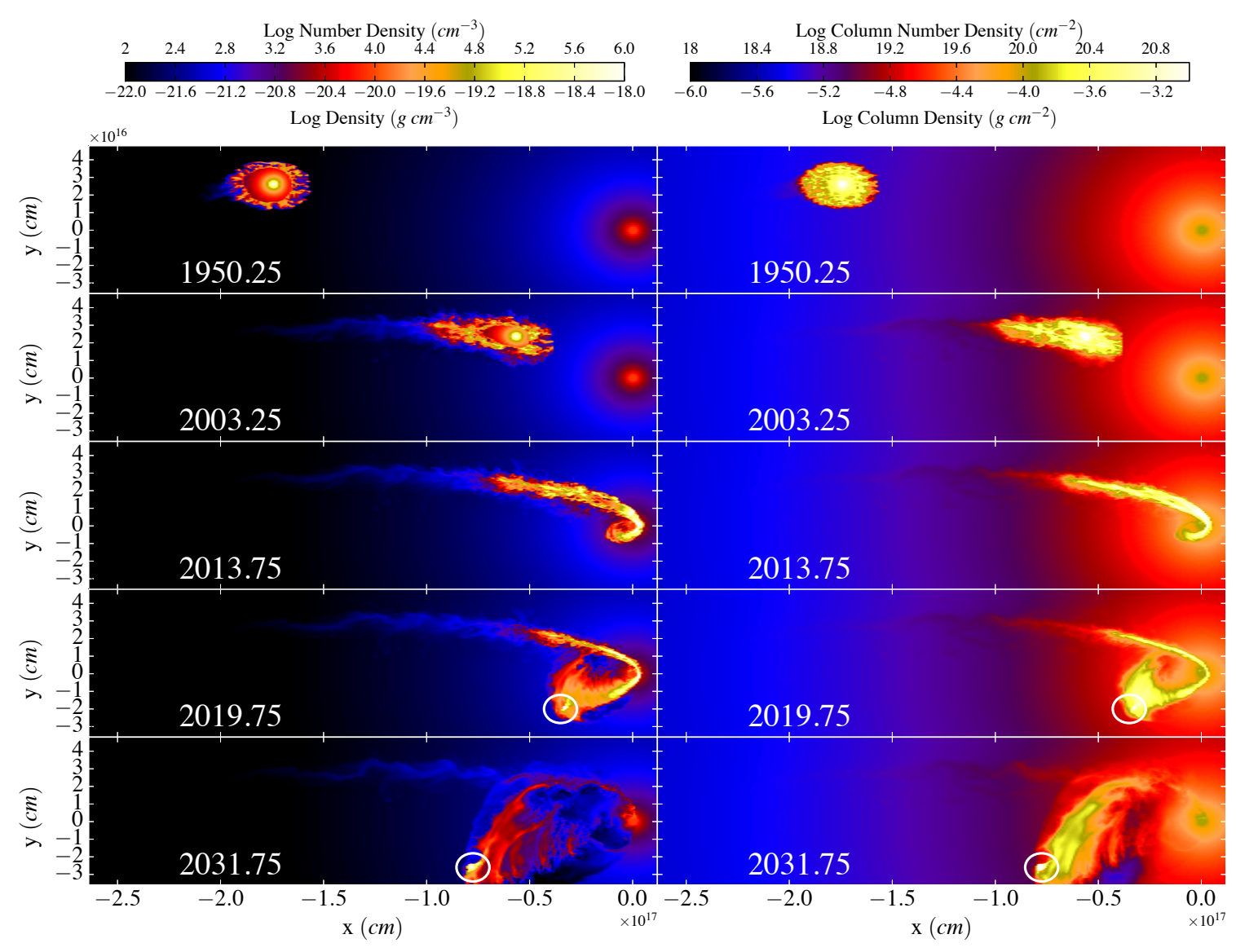

Figure 1. Density maps for the standard model. Left panels show the density distribution in a slice at $\mathrm{z}=0$. The right panels show the column density, i.e. the integral of the density along the $\mathrm{z}$ direction. The white circles show the outflow reforming after pericentre.

$\mathrm{CHOMBO}^{1}$ library. For the refinement criterion, we chose the standard one in PLUTO, based on the second derivative error norm, and we applied it to the density. The criterion has been widely tested and it is able to resolve most of G2's material at the highest resolutions. The computational do-

1 https://seesar.lbl.gov/anag/chombo/ main is Cartesian (with the exception of one test run in $2 \mathrm{D}$ cylindrical coordinates, see Table 1). A two-shock Riemann solver (Mignone et al. 2012) has been chosen for the solution of the hydrodynamic equations.

The outflow is modeled in a "mechanical" way as in Ballone et al. (2013, 2016) and De Colle et al. (2014), where 
the velocity is set to the constant wind value $v_{\mathrm{w}}$ and the density $\rho_{\mathrm{w}}$ is set to satisfy

$\dot{M}_{\mathrm{w}}=4 \pi r_{\mathrm{w}}^{2} \rho_{\mathrm{w}} v_{\mathrm{w}}$.

In order to reach a reasonable sampling of the input region, i.e. a good isotropy of the outflow, the input region's radius $r_{\mathrm{w}}$ is varying, in time, proportional to the theoretical stagnation radius $R_{\text {out }}$ (see Eq. 8), with minimum and maximum allowed values equal to $2.10 \times 10^{14} \mathrm{~cm}$ and $1.05 \times 10^{15} \mathrm{~cm}$, respectively. The temperature of the injected material is set to $T_{\mathrm{w}}=10^{4} \mathrm{~K}$ and an adiabatic index $\Gamma=1$ has been assumed (see discussion in Ballone et al. 2013).

Compared to Ballone et al. (2013), the source's orbit is now a proper elliptical orbit and it has been updated to the one derived by Gillessen et al. (2013b) through Br $\gamma$ observations. The orbit has been previously integrated with a leapfrog method and the source's positions and velocities are interpolated from the stored ones using a 1st order Newton polynomial formula.

The hot atmosphere is modeled following the density and temperature distribution used by several authors (see Burkert et al. 2012; Schartmann et al. 2012; Anninos et al. 2012; Ballone et al. 2013; De Colle et al. 2014; Ballone et al. 2016), i.e.,

$n_{\mathrm{at}} \simeq 5.60 \times 10^{3}\left(\frac{1}{d_{\mathrm{BH}, \text { peri }}}\right) \mathrm{cm}^{-3}$,

$T_{\text {at }} \simeq 7.12 \times 10^{8}\left(\frac{1}{d_{\mathrm{BH}, \text { peri }}}\right) \mathrm{K}$,

where $d_{\mathrm{BH} \text {,peri }}$ is the distance from $\mathrm{Sgr} \mathrm{A}^{*}$ in units of the pericentre distance, i.e. $3 \times 10^{15} \mathrm{~cm}$.

This is a very idealized model and, given the uncertainties in the actual distribution of the accretion flow around SgrA*, we still decided to keep it as idealized as possible, to be able to better understand 0th-order hydrodynamical effects on G2. This would be difficult when doing more sophisticated modeling. As in Schartmann et al. (2012, 2015) and Ballone et al. (2013,2016), we reset the atmosphere with the help of a passive tracer. In order to reproduce the outer shock propagating in the atmosphere, De Colle et al. (2014) did not apply the same recipe for two of their simulations. However, in these cases, the development of convective bubbles all around the SMBH region is apparent. This artifact is avoided in our approach. Finally, the SMBH's gravitational field has been modeled as a Newtonian point source with mass $M_{\mathrm{BH}}=4.31 \times 10^{6} M_{\odot}$ (Gillessen et al. 2009) at $x, y, z=0$. We refer to Ballone et al. (2013) for further discussions and details about the modeling and the assumptions.

As in Ballone et al. (2013, 2016) and differently than in De Colle et al. (2014), we decided to start the simulation (and the outflow) at apocentre. As already pointed out in Ballone et al. (2013), this choice is somehow arbitrary. However, if the source of G2 had been scattered via multiple encounters (Murray-Clay \& Loeb 2012) from the clockwise rotating disk of young stars (Paumard et al. 2006; Bartko et al. 2009), any pre-existing gas envelope would have been tidally torn apart.
A list of the simulations discussed in the present paper can be found in Table 1 .

\section{RESULTS}

The purpose of this section is to present the evolution of our new 3D simulations and their comparison to observations, in which we will focus on the new and accurate construction of mock PV diagrams and on the interpretation of the time dependence of the total Brackett- $\gamma$ luminosity.

\subsection{The standard model}

We adopt a mass loss rate of $\dot{M}_{\mathrm{w}}=5 \times 10^{-7} \mathrm{M}_{\odot} \mathrm{yr}^{-1}$ and a wind velocity of $v_{\mathrm{w}}=50 \mathrm{~km} \mathrm{~s}^{-1}$ for our standard model.

As shown in Fig. 1, the evolution of the density distribution in this $3 \mathrm{D}$ simulation is very similar to the one of the 2D simulations in Ballone et al. (2013) (for an exhaustive discussion on the physics of these winds, we also refer the reader to Christie et al. 2016; Ballone et al. 2016). The outflow is free-flowing until its ram pressure reaches the pressure of the external hot and dense atmosphere. Hence, it is composed of an inner part, whose density scales as $1 / r^{2}$ (due to the continuity equation), that is surrounded by the part of the outflow that gets shocked by the impact with the atmosphere. This shocked material is highly RayleighTaylor unstable. At the beginning, the outflowing material is still in a quasi-spherical configuration, since the isotropic thermal pressure of the atmosphere is still dominant compared to the anisotropic ram pressure. At later times, the free-wind region shrinks due to the increasing thermal pressure, the ram pressure makes it asymmetric and the stripped shocked material is forming a small tail trailing the source. Overall, though more filamentary, the distribution of the outflowing gas is on large scales very similar to the one in the "diffuse cloud" simulations of Schartmann et al. (2012), Anninos et al. (2012) and Schartmann et al. (2015), particularly right before and after pericentre, when the material is first compressed into a thin filament by the tidal force from the SMBH and then expands, strongly increasing its cross section.

Due to the asymmetry of the free-wind region and the formation of the small tail of stripped material, at the time of the observations, the central source is always in the leading part of G2. The immediate implication is that the photocentre of the emission will never be on top of the source.

As expected, the simulation also shows that the emitting source becomes, at a certain point, distinguishable from the rest of G2. This might happen already around year 20192020 , when the source creates a second peak in the density distribution (see circles in the lowermost panels of Fig. 1). This is a clear difference compared to the diffuse cloud simulations and the decoupling between the source and the previously emitted gas, after pericentre, could eventually be the smoking gun to understand the nature of G2.

\subsection{Matching the PV diagrams}

Compared to Ballone et al. (2013), the 3D simulation now allows us to construct realistic PV diagrams, like the ones already presented in Schartmann et al. (2015). To do this, 

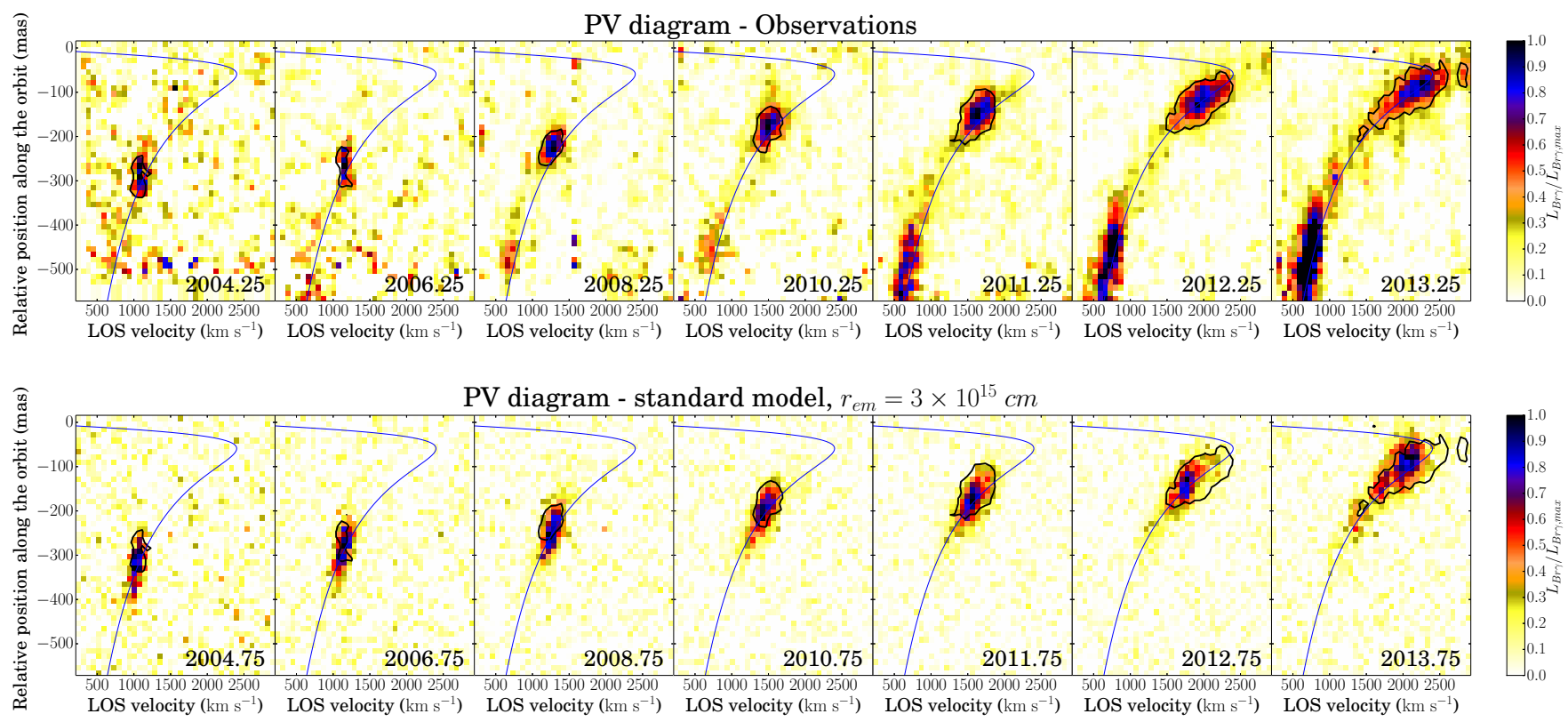

Figure 2. Position-Velocity diagrams for the standard model. The upper panel shows the observations, while the lower one shows the case of $r_{\mathrm{em}}=3 \times 10^{15} \mathrm{~cm}$. The black contours show the position and extent of the observed G2.

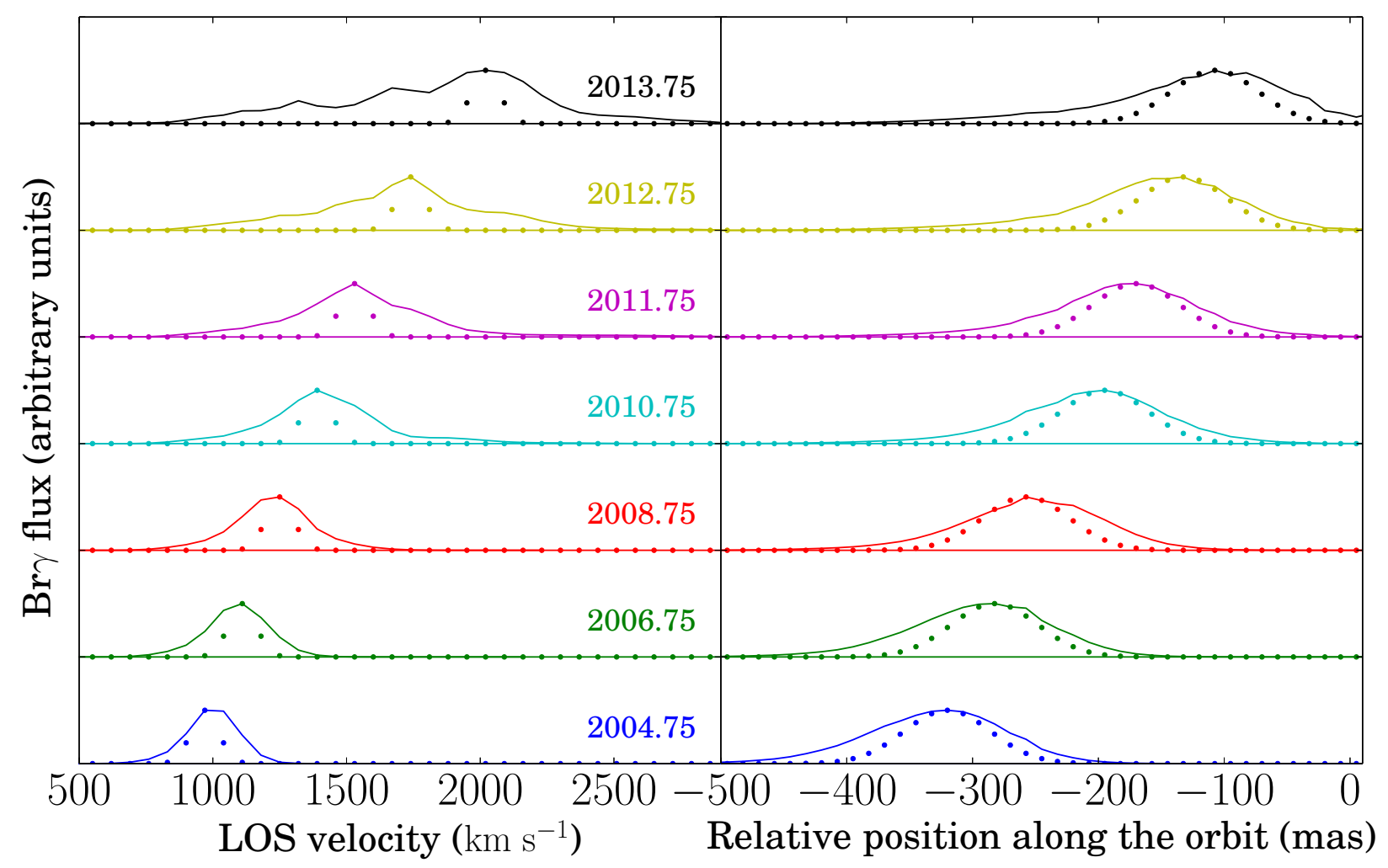

Figure 3. Line width (left) and size along the orbit (right) evolution for the standard model, in the case of $r_{\mathrm{em}}=3 \times 10^{15} \mathrm{~cm}$ (solid lines). The dots represent a gaussian distribution centred on the peak of the emission, with FWHM equal to $120 \mathrm{~km} \mathrm{~s}^{-1}$ in velocity and 81 mas in the size, corresponding to the spectral and spatial point spread function of SINFONI. 
we first project every cell in our computational domain onto the sky plane, according to the last orbital elements derived by Gillessen et al. (2013b) for the $\operatorname{Br} \gamma$ observations. This is done through a transformation from $\left(x, y, z, v_{x}, v_{y}, v_{z}\right)$ to ( $r a$, decl, losv) where $r a$, decl and losv are the right ascension, the declination and the line of sight (l.o.s.) velocity, respectively. We can, from this, create a $3 \mathrm{D}$ histogram of the $\mathrm{Br} \gamma$ luminosity, with bin size equal to 12.5 mas for $r a$ and decl and $69.6 \mathrm{~km} \mathrm{~s}^{-1}$ for losv. These values correspond to the size of the 3D pixels (voxels) in a SINFONI data cube. We then apply a smoothing in all directions with FWHM equal to 81 mas in right ascension and declination and to 120 $\mathrm{km} \mathrm{s}^{-1}$ in l.o.s. velocity. These values correspond to the spatial point spread function (PSF) and spectral resolution. At this point, every cell is spatially projected onto the derived orbit, using it as a curved slit in the ( $\mathrm{ra}$, decl) space (a slit curved along G2's orbit has also been used for the construction of the observed PV diagrams; see Gillessen et al. 2013a). The former operation reduces the triplet ( $r a$, decl, losv) to a couple (pos, losv), where pos is the position on the orbit, and creates a 2D position-velocity histogram. Given the uncertainties in the luminosity discussed in Sec. 4.1 and 4.2, every PV diagram is then scaled to its maximum. Noise is finally extracted from the observed PV diagrams and added to the simulated ones.

The luminosity is calculated using a functional form for the case $\mathrm{B}$ recombination $\mathrm{Br} \gamma$ emissivity

$j_{\mathrm{Br} \gamma}=3.44 \times 10^{-27}\left(T / 10^{4} \mathrm{~K}\right)^{-1.09} n_{\mathrm{i}} n_{\mathrm{e}} \mathrm{erg} \mathrm{s}^{-1} \mathrm{~cm}^{3}$,

(where $T$ is the wind material temperature and $n_{\mathrm{i}}$ and $n_{\mathrm{e}}$ are the ion and electron number densities), obtained by extrapolating the values given on page 73 in Osterbrock \& Ferland (2006) (see also Ferland 1980; Hamann \& Ferland 1999; Ballone et al. 2013).

In Sec. 4.1 we will show that the amount of emission coming from the free flowing part of the outflow is uncertain. For an outflow scenario, this is strongly dependent on the flux of ionizing photons reaching G2, which is not exactly constrained. For this reason, we present here the effect of different contributions on the total $\mathrm{Br} \gamma$ luminosity of the free flowing region. Namely, we calculate PV diagrams assuming that the latter is ionized and emits in $\mathrm{Br} \gamma$ only up to a certain inner radius $r_{\mathrm{em}} \cdot r_{\mathrm{em}}$ is hence a free parameter of our post-processing and we choose $r_{\mathrm{em}}=\left[3 \times 10^{14}, 10^{15}, 3 \times\right.$ $10^{15} \mathrm{~cm}$. We also calculated PV diagrams for the shocked outflow material only (in the text we will denote this case with $\left.r_{\mathrm{em}}=r_{\text {shock }}\right)$.

The results are shown in Fig. 2, 3 and 4. Due to the particularly dramatic evolution of the gas during and after pericentre, the already mentioned issues related to the luminosity discussed in Sec. 4.1 and 4.2 are significantly effecting the reliability of our mock PV diagrams after 2014.5. For this reason, we restrict our comparison to the pre-pericentre part of the orbit and avoid making predictions for post-pericentre PV diagrams. As mentioned in Sec. 3.1, the orbital solution of the centre of the emission is never equal to that of the source; our comparison uses a time offset of roughly half a year between simulation and observations. The match is not perfect. In principle, a quantitative comparison between observed and simulated PV diagrams might eventually be used, through an iterative repetition of the simulation, to
PV diagram - standard model, $r_{e m}$ study

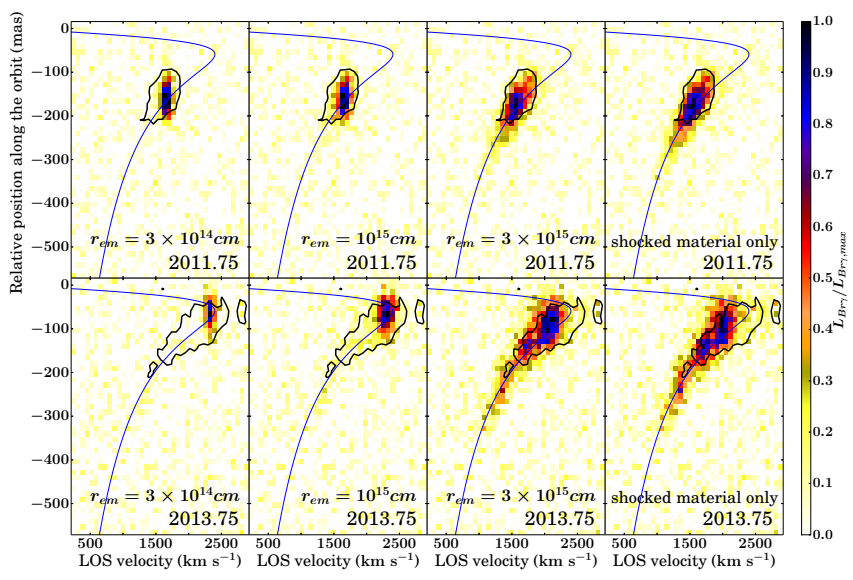

Figure 4. Position-Velocity diagrams for the standard model. The different panels show the simulated PV diagrams for different assumptions on the inner emitting radius $r_{\mathrm{em}}$. For every panel, the luminosity per bin is scaled to the maximum one. The upper and lower panels are obtained for a simulation year of 2011.75 and 2013.75, respectively. The black contours show the position and extent of the observed G2.

determine the orbit of the source that produces the perfect match. However, the high number of parameters of our models might not allow a strong constraint of the source's orbit and the high computational cost of these hydrodynamic simulations does not allow such a numerical experiment. Our purpose is rather to show which mass-loss rates and velocities an outflow should have to produce a reasonable result. This is already not trivial. Hence, we stick to a qualitative comparison and use a simple constant time offset. We must stress, thus, that this offset has no strong quantitative meaning. Nonetheless, as visible in Fig. 2 and 3, our standard case is able to reproduce an increase in the line-width of the $\mathrm{Br} \gamma$ emission, as in the case of the observations of G2. These figures also show that our standard model appears marginally resolved, even with some broadening of G2's size with its pericentre approach, as shown by Gillessen et al. (2013b) and Pfuhl et al. (2015).

When looking at the $r_{\mathrm{em}}=r_{\text {shock }}, 3 \times 10^{15} \mathrm{~cm}$ cases, the simulated material has a qualitatively comparable extent, even though it fails to reproduce the high velocity material that seems to overshoot the orbit derived from observations, just before the pericentre passage. This is again due to the fact that, close to pericentre, the outflow material is asymmetrically distributed with respect to the source, with most of the material in a trailing tail. When going to smaller values of $r_{\mathrm{em}}$, the emitting region moves to slightly higher velocities and positions on the orbit, but it becomes smaller and smaller in the PV diagrams. This is a direct consequence of the location and of the important impact of the free-wind region on the outflow emission. In fact, given Eq. 1, the emission measure $\mathrm{EM} \propto \int \rho_{\mathrm{w}} d V \propto r^{-1}$ is diverging for small distances from the source. As a result, the more the inner part of the free-wind region is included, the more dominant the free-wind region, the smaller the emitting region visible in the PV diagrams. 

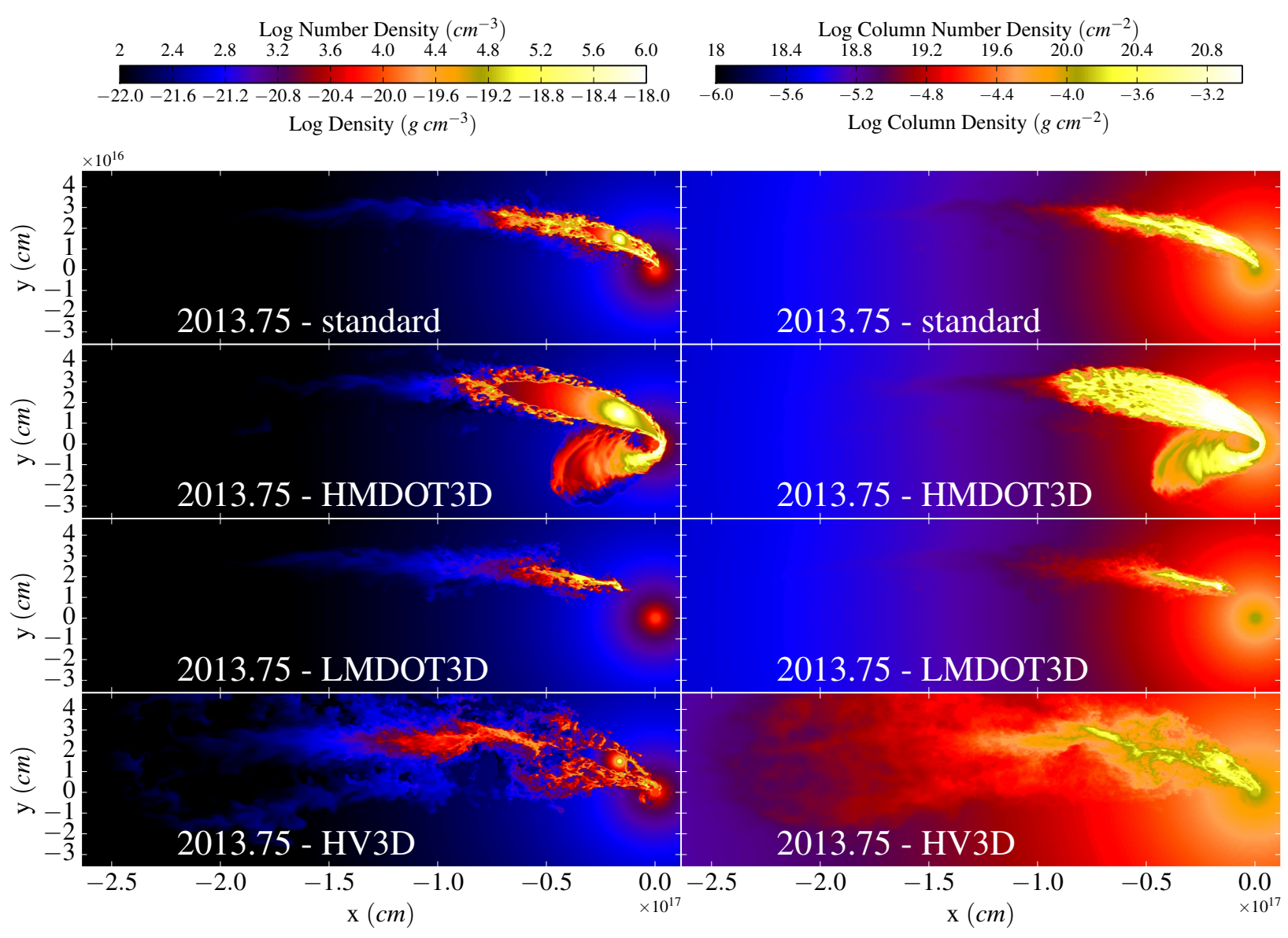

Figure 5. Density maps for the simulations of our parameter study. Left panels show the density distribution in a slice at $\mathrm{z}=0$. The right panels show the column density, i.e. the integral of the density along the $\mathrm{z}$ direction.

So, all in all, we conclude that a good match with the observations can be reached only if a tiny fraction of the free-wind region is actually emitting. This conclusion is general and can be also deduced from the parameter study in Sec. 3.3, where we show that G2 appears too small for every model, when $r_{\mathrm{em}}<3 \times 10^{15} \mathrm{~cm}$. A probably better result could also be reached with a slightly different (more eccentric) orbital solution. In fact, uncertainties in the observations seem to give enough room for this possibility. However, testing it directly with simulations is beyond the scope of the present work.

\subsection{Parameter study}

Following Ballone et al. (2013), we performed a parameter study, varying the mass-loss rate and the velocity of the outflow. We hence run models LMDOT3D and HMDOT3D with the same velocity as the standard model's one, but with a factor of 5 smaller and larger mass-loss rate, respectively. Concerning the velocity, we chose to run just the HV3D model, with wind velocity equal to $v_{\mathrm{w}}=250 \mathrm{~km} \mathrm{~s}^{-1}=$ $5 \times v_{\mathrm{w}, \text { standard }}$. As already discussed in Ballone et al. (2013), given the isothermal equation of state, a temperature of $T=10^{4} \mathrm{~K}$ in the injected material brings the sound speed of the wind to $c_{\mathrm{s}, \mathrm{w}} \approx 10 \mathrm{~km} \mathrm{~s}^{-1}$. As a consequence, for wind velocities too close to $c_{\mathrm{s}, \mathrm{w}}$, the injected thermal and ram pressure become comparable, leading to too high mass loss rates and velocities. However, Ballone et al. (2013) have already shown that a lower outflow velocity has the effect of reducing G2's size.

Fig. 5 shows the density maps for the three models of the parameter study. As already described in Ballone et al. (2013) and Ballone et al. (2016), for LMDOT3D and HMDOT3D the outflow is too dense for the ram-pressure stripping to be efficient enough. Hence, the size of the outflow is mainly given by momentum equilibrium between the outflow and the external forces, namely the thermal and ram pressures of the atmosphere and the tidal force of the SMBH. This explains why LMDOT3D and HMDOT3D are respectively smaller and bigger than the standard model. In the HV3D case, the outflow is much less dense and the shocked material spreads out over a large volume. This enables the formation of a long cometary tail by efficient ram pressure stripping, as in the case of the model in Ballone et al. (2016). 
Fig. 6 shows the PV diagrams for our parameter study. In the case of model HMDOT3D, G2 looks too elongated when only the shocked wind material is considered, while a reasonable match to observations could eventually be reached in the case of $r_{\mathrm{em}}>3 \times 10^{15} \mathrm{~cm}$. Model LMDOT3D is instead producing a too compact emission for every assumption on $r_{\mathrm{em}}$. HV3D can instead result in a bimodal distribution in the PV diagrams, when looking at the emission of the shocked material only. For HV3D, the separation between the two simulated emission spots is not large enough to match the observed position of G2 and G2t on the orbit (see Fig. 2), but motivated our attempt to model both components with a single wind source (Ballone et al. 2016).

When looking at the luminosity evolution in Fig. 7, while models LMDOT3D and HV3D have a too low luminosity (roughly confirming the trends found in Ballone et al. 2013), model HMDOT3D is matching the observations when the shocked-material only is considered, while it is a factor $\approx 2$ too luminous when $r_{\mathrm{em}}=3 \times 10^{15} \mathrm{~cm}$ is adopted. The first evident effect is that lower mass-loss rates or higher velocities produce globally lower luminosities. This is simply explained by Eq. 1 and 4, showing that the luminosity is proportional to the integral of $\rho_{\mathrm{w}}^{2}$. and that $\rho_{\mathrm{w}}$ is directly proportional to the mass-loss rate and inversely proportional to the outflow velocity. So, on a 0 -th order, outflows with lower mass-loss rates and/or higher velocities are less dense (even in their shocked part) and have a lower emission measure, and vice versa. For any fixed model, a varying contribution is also given by the free-wind region, depending on the choice of $r_{\mathrm{em}}$. This result, however, is in contradiction with what has been found by Ballone et al. (2013) with 2D simulations, where the shocked material was dominating the total luminosities close to pericentre. This is mainly explained by the poor resolution of the present simulations, as discussed in Sec. 4.2. As a consequence, we conclude that the absolute values of the calculated luminosities must be taken as lower limits, while the structure in the PV diagrams is a more solid and stable diagnostic tool.

\section{DISCUSSION}

For a critical interpretation of the results presented in the previous section, a discussion of a few issues concerning the calculation of the $\mathrm{Br} \gamma$ luminosity is needed.

The first issue is clearly visible in Fig. 4 and 6: in order for this scenario to reproduce the size of G2 in the positionvelocity space, most of the inner unperturbed part of the outflow must be neutral (hence, dark in recombination line emission; $\left.r_{\mathrm{em}} \gtrsim 3 \times 10^{15} \mathrm{~cm}\right)$. Understanding whether this is actually the case would require a solid knowledge and treatment of the source of gas ionization. A full integration of radiation in the hydrodynamical simulation is needed, but the current simulations were already extremely time consuming and a further coupling with a radiative transfer scheme would make them unfeasible. On top of this, however, most of the available studies make use of simplified and (sometimes dramatically) different assumptions on the flux of ionizing photons reaching G2. In section 4.1 we present a very basic calculation of the amount of free-wind region that is actually ionized by Lyman-continuum (Lyc) photons from the surrounding stars. Such estimate contains several

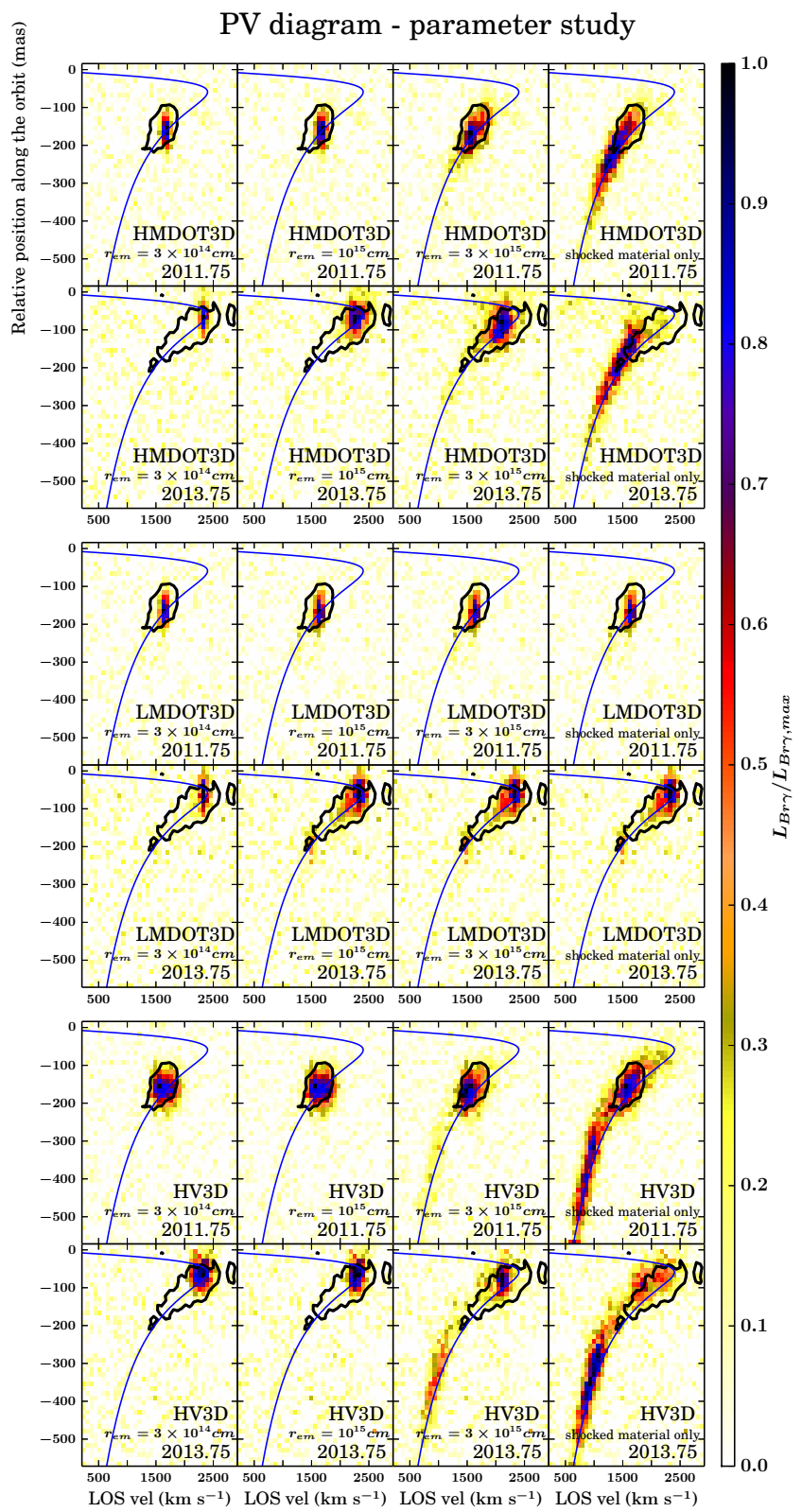

Figure 6. Position-Velocity diagrams for our parameter study. The different panels show the simulated PV diagrams for different assumptions on the inner emitting radius $r_{\mathrm{em}}$. For every panel, the luminosity per bin is scaled to the maximum one. The black contours show the position and extent of the observed G2.

caveats, hence it does not have particularly strong physical basis; nonetheless, it clearly shows how the contribution of the free-flowing region can be severely dependent on the different assumptions on the Lyc photons flux on G2.

Another issue concerns the effect of numerics in the simulations on the resulting $\operatorname{Br} \gamma$ luminosity and, particularly, on the luminosity curves in Fig. 7. We show in section 4.2 that the absolute value of the total luminosity can be significantly affected by the resolution and by the geometry and symmetry used for the simulation. From this, we can conclude that the PV diagrams are more stable diagnostics, 


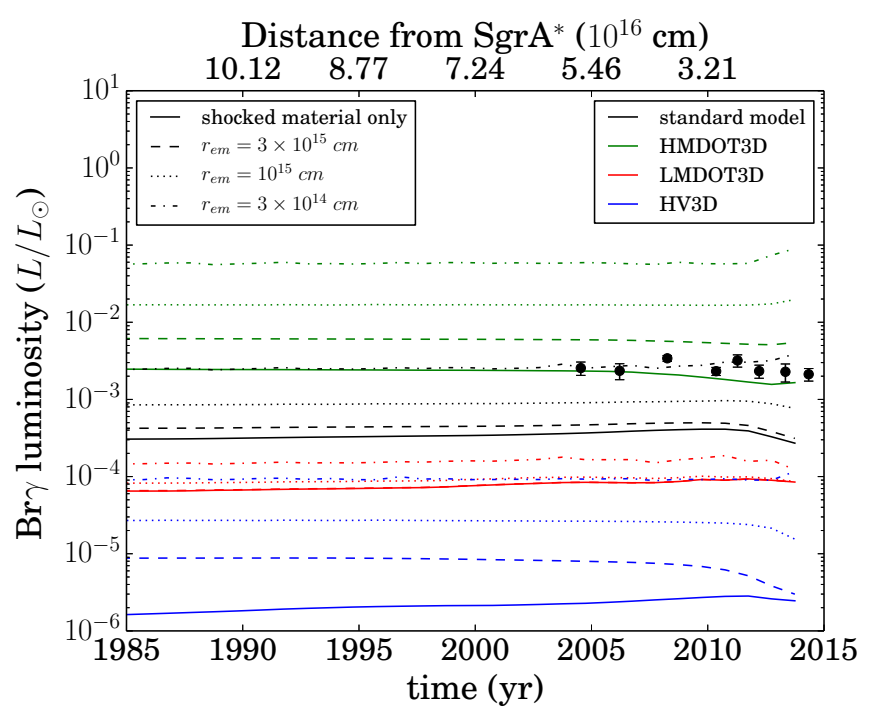

Figure 7. Luminosity comparison for our simulations. The different colours show different models, the different linestyle refers to different assumptions for the inner emitting radius $r_{\mathrm{em}}$. The black points show the luminosities observed by Pfuhl et al. (2015).

compared to luminosity curves, since they essentially represent the relative contribution to the total luminosity from different regions of G2.

After such needed discussions, the second part of this section tries to compare our work to what has been done by other authors (section 4.3) and to give our model a more physical context, with a focus on the possible nature of the central source (section 4.4) and of the advantages and disadvantages of this scenario, compared to the "diffuse cloud" one (section 4.5).

\subsection{Ionization of the outflow}

In this section we try to estimate the contribution to the total luminosity of the free-flowing region of any wind in the Galactic Centre. The following calculation is based on the assumption that the ionization of the gas fully comes from UV photons from the nearby young stars (see Section 1 and Gillessen et al. 2012). Unfortunately, the flux of ionizing photons reaching G2 is not well known, so we decided to stick to a very simplified analytical calculation (see section 4.1.1 for a discussion about its limitations). Its main purpose consists in justifying the need of $r_{\mathrm{em}}$ as a free parameter in the analysis of our hydrodynamic simulations.

The derivation is based on equating, in a one dimensional fashion, the rate of UV ionizing (Lyc) photons isotropically penetrating a spherical ("naked" free-flowing) region, whose density scales as $1 / r^{2}$, to the rate of recombinations occurring within the latter. In this way, we get the number of atoms in a free-wind shell needed to "consume" all the ionizing photons reaching G2. The thickness of this shell is depending on the total size of the free-wind region and, of course, on the amount of available Lyc photons.
The inferred equation is

$\phi\left(\frac{R_{\mathrm{out}}}{D}\right)^{2} \approx \int_{R_{\mathrm{in}}}^{R_{\mathrm{out}}} \alpha_{\mathrm{rec}} n_{\mathrm{e}} n_{\mathrm{i}} 4 \pi r^{2} d r$

where $\phi$ is the rate of emitted ionizing photons and $D$ is the distance of G2 from the ionizing source. However, the value of these two latter quantities is not very well constrained and one must assume there is more than one emitting source. In the following, $\phi / 4 \pi D^{2}$ will simply be the flux of ionizing photons on G2 and we will consider different numbers used in previous calculations by different authors. $R_{\text {out }}$ and $R_{\text {in }}$ are respectively the outer and inner radius of the ionized shell. $\alpha_{\text {rec }}$ is the total recombination coefficient and we assumed $\alpha_{\mathrm{rec}}=2.59 \times 10^{-13} \mathrm{~cm}^{3} \mathrm{~s}^{-1}$, i.e. the value for case $B$ recombination for pure hydrogen at $T=10^{4} \mathrm{~K}$ (Osterbrock \& Ferland 2006, page 22). $n_{\mathrm{e}}$ and $n_{\mathrm{i}}$ are the number densities of the electrons and ions - respectively in the gas and $r$ is the distance from the source. For a $1 / r^{2}$ density profile,

$n_{\mathrm{e}} n_{\mathrm{i}} \approx \frac{\rho^{2}}{\mu_{\mathrm{e}} \mu_{\mathrm{i}} m_{\mathrm{H}}^{2}} \approx \frac{\dot{M}_{\mathrm{w}}^{2}}{16 \pi^{2} v_{\mathrm{w}}^{2} \mu_{\mathrm{e}} \mu_{\mathrm{i}} m_{\mathrm{H}}^{2} r^{4}}$,

where $\dot{M}_{\mathrm{w}}$ and $v_{\mathrm{w}}$ are the mass-loss rate and velocity of the wind, respectively, $\mu_{\mathrm{e}}=1.17$ and $\mu_{\mathrm{i}}=1.29$ are the electron and ion mean weight (for solar metallicity) and $m_{\mathrm{H}}$ is the hydrogen mass.

So, substituting $n_{\mathrm{e}} n_{\mathrm{i}}$ in Eq. 5 and solving the integral, we can get the inner radius $R_{\text {in }}$ for which there is a balance between the rate of incoming ionizing photons and the rate of recombinations, over the whole volume:

$R_{\text {in }}=\left[\frac{\phi}{D^{2}} \frac{4 \pi v_{\mathrm{w}}^{2} \mu_{\mathrm{e}} \mu_{\mathrm{i}} m_{\mathrm{H}}^{2} R_{\mathrm{out}}^{2}}{\alpha_{\mathrm{rec}} \dot{M}_{\mathrm{w}}^{2}}+\frac{1}{R_{\text {out }}}\right]^{-1}$.

The total volume of the free-wind region changes as the source moves along the orbit and encounters a higher and higher external pressure. In this case, we assume that the outer radius $R_{\text {out }}$ is just the stagnation radius given by the atmosphere's thermal pressure only (we hence neglect any anisotropic pressure contributions)

$R_{\text {out }}=\left[\frac{\dot{M}_{\mathrm{w}} v_{\mathrm{w}}}{4 \pi P_{\mathrm{amb}}}\right]^{1 / 2}$.

Substituting Eq. 8 in Eq. 7, we get

$R_{\text {in }}=\left[\frac{\phi}{D^{2}} \frac{v_{\mathrm{w}}^{3} \mu_{\mathrm{e}} \mu_{\mathrm{i}} m_{\mathrm{H}}^{2}}{\alpha_{\mathrm{rec}} \dot{M}_{\mathrm{w}} P_{\mathrm{amb}}}+\left(\frac{4 \pi P_{\mathrm{amb}}}{\dot{M}_{\mathrm{w}} v_{\mathrm{w}}}\right)^{1 / 2}\right]^{-1}$.

For our choice of the atmosphere (see Eq. 2 and 3) the ambient thermal pressure is varying with radius and so will the inner and outer radii do:

$$
\begin{aligned}
R_{\mathrm{in}} & \approx 3 \times 10^{15}\left[1.691 \times 10^{-2} \frac{\phi_{50}}{D_{\mathrm{pc}}^{2}} \frac{v_{\mathrm{w}, 50}^{3} d_{\mathrm{BH}, \text { peri }}^{2}}{\dot{M}_{\mathrm{w},-7}}+\right. \\
& \left.+44.099\left(\frac{1}{\dot{M}_{\mathrm{w},-7} v_{\mathrm{w}, 50}}\right)^{1 / 2} \frac{1}{d_{\mathrm{BH}, \text { peri }}}\right]^{-1} \mathrm{~cm}
\end{aligned}
$$

where we expressed the rate of ionizing photons in units 
of $10^{50} \mathrm{~s}^{-1}, \mathrm{D}$ in units of pc, the wind's mass-loss rate $\dot{M}_{\mathrm{w}}$ in units of $10^{-7} M_{\odot} \mathrm{yr}^{-1}$, its velocity $v_{\mathrm{w}}$ in units of $50 \mathrm{~km} \mathrm{~s}^{-1}$ and the distance from $\mathrm{SgrA}^{*} d_{\mathrm{BH}, \text { peri }}$ in units of the pericentre distance.

We performed the calculation for $v_{\mathrm{w}}=50 \mathrm{~km} \mathrm{~s}^{-1}$ and $\dot{M}_{\mathrm{w}}=10^{-7}, 10^{-6} M_{\odot} \mathrm{yr}^{-1}$, assuming five different fluxes of UV photons:

- In the first case, which we will call SB, we assumed the numbers used by Scoville \& Burkert (2013), i.e. $\phi_{50}=1$ and $D_{\mathrm{pc}}=1$. This assumption is equivalent to having a single O5 star at a constant distance of $1 \mathrm{pc}$.

- In the second case, MLlow, we took numbers from Murray-Clay \& Loeb (2012) for the entire central parsec; these numbers are (more or less) matching the values provided in Martins et al. (2007) and Genzel et al. (2010). In particular, they assume $\phi_{50}=10^{0.8} \simeq 6.31$ for $D_{\mathrm{pc}}=1$. This is a lower estimate for the UV flux given by these authors.

- Murray-Clay \& Loeb (2012) also took into account the concentration of the S-stars (of spectral class B) within the very central region of the Galactic Centre. They estimate these stars to produce a total $\phi_{50}=0.2$, but for a region of $D_{\mathrm{pc}} \simeq 6 \times 10^{-3}$. This is their higher estimate and we refer to it as MLhigh.

- In the fourth case, Sh04, we assumed the flux used by Shcherbakov (2014) for the position of the cloud in the year 2004. We consider the values derived by this author as the most reasonable ones, since they are obtained calculating the contribution of the main Wolf-Rayet stars in the young cluster, exactly taking into account their positions, from Paumard et al. (2006) and Lu et al. (2009), and their temperatures and luminosities, from Martins et al. (2007). In $2004, F_{\mathrm{UV}}=3 \times 10^{4} \mathrm{erg} \mathrm{s}^{-1} \mathrm{~cm}^{-2}$. If we crudely divide this value by the ionization energy of the hydrogen atom, we get the number flux of ionizing photons $\phi_{50} / D_{\mathrm{pc}}^{2} \simeq 131$.

- In the last case, Sh14, we assumed the flux assumed by Shcherbakov (2014) at G2's pericentre, namely $F_{\mathrm{UV}}=5.7 \times$ $10^{4} \mathrm{erg} \mathrm{s}^{-1} \mathrm{~cm}^{-2}$. Close to pericentre, the flux increases due to the contribution of the star S2. Dividing by the ionization energy of the hydrogen atom, we get $\phi_{50} / D_{\mathrm{pc}}^{2} \simeq 249$.

In Fig. 8 we plot the results of our simple analytical calculation. As visible in the central panel, the size of $R_{\text {in }}$ first increases with G2 getting closer to SgrA* and then decreases at smaller distances. This is the result of two competing effects, i.e. the decrease of available ionizing photons and the increasing density (and number of recombinations) in the outer layer of the free-wind region with the shrinking of the stagnation radius. These two different branches are mathematically visible in Eq. 7 and 10, as asymptotic branches $\propto R_{\text {out }}^{-2} \propto d_{\mathrm{BH}}^{-2}$ for large distances and $\propto R_{\text {out }} \propto d_{\mathrm{BH}}$ for small ones (see also the upper panel in Fig. 8). It is also interesting to note that the transition between these two branches moves to larger values of $d_{\mathrm{BH}}$ for smaller values of $\phi / D^{2}$. On the other hand, the lower panel of Fig. 8 shows that the $\mathrm{Br} \gamma$ luminosity is a monotonic function of $R_{\text {out }}$ and $d_{\mathrm{BH}}$. This is easily understandable from Eq. 5: as the $\mathrm{Br} \gamma$ luminosity is directly proportional to the number of recombinations (i.e., the right hand side of the equation), it is also $\propto R_{\text {out }}^{2}$.

As just described, the evolution of $R_{\text {in }}, R_{\text {in }} / R_{\text {out }}$ and the $\mathrm{Br} \gamma$ luminosity, as a function of the distance from the black hole is a direct result of the previous equations, hence

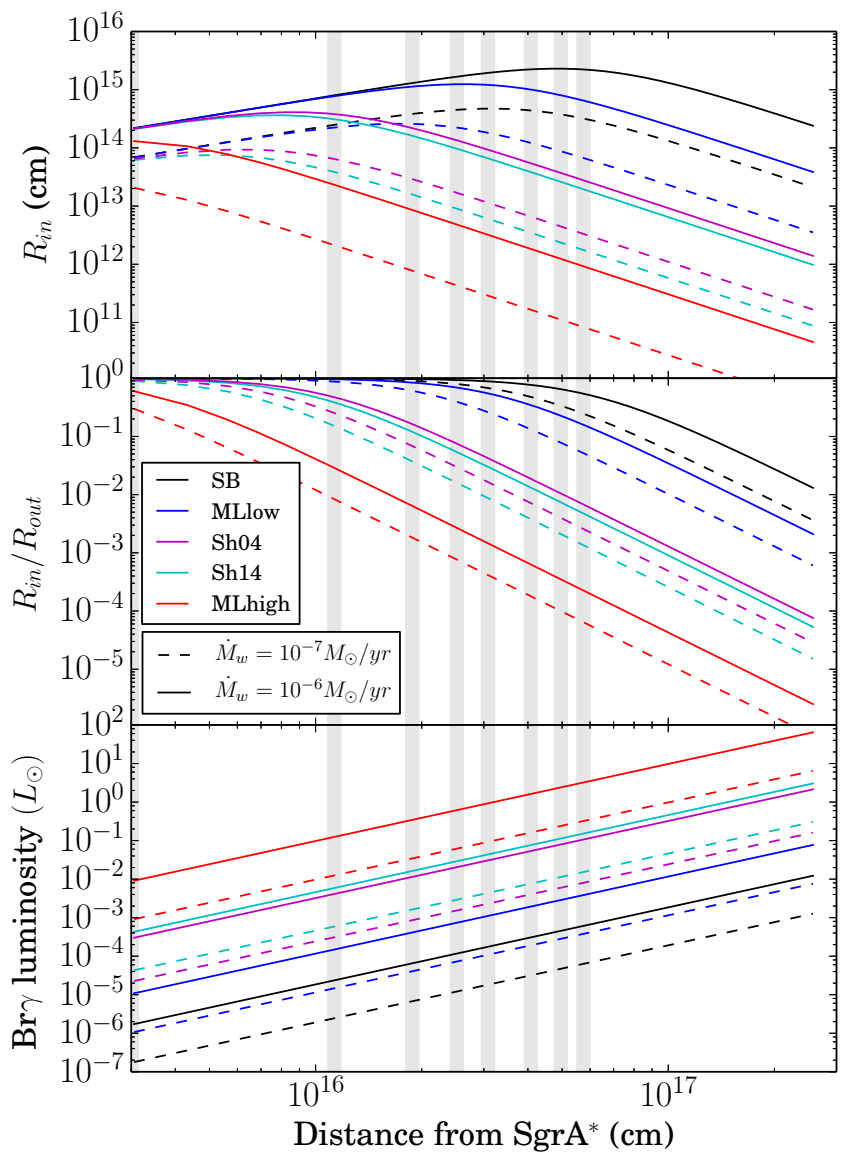

Figure 8. Results of our analytical calculation for the ionization of the free-wind region discussed in Sec. 4.1. The upper panel shows the absolute value of the inner radius $R_{\text {in }}$, while the central panel shows the ratio between the inner $\left(R_{\text {in }}\right)$ and outer $\left(R_{\text {out }}\right)$ radii of the ionized shell. The luminosity of the spherical freeflowing ionized shell is plotted in the lower panel. The dashed and solid lines show the results for $\dot{M}_{\mathrm{w}}=10^{-7}, 10^{-6} M_{\odot} \mathrm{yr}^{-1}$, respectively (the wind velocity is $v_{\mathrm{w}}=50 \mathrm{~km} \mathrm{~s}^{-1}$ for both calculations). Different colours show the results for different assumptions on the flux of ionizing photons in the Galactic Centre: $\left(\phi / D^{2}\right)_{\mathrm{SB}}=1,\left(\phi / D^{2}\right)_{\text {MLlow }}=6.31,\left(\phi / D^{2}\right)_{\mathrm{Sh} 04}=131$, $\left(\phi / D^{2}\right)_{\mathrm{Sh} 14}=249,\left(\phi / D^{2}\right)_{\text {MLhigh }}=5560$. The grey vertical bands correspond to $d_{\mathrm{BH}}$ of $\mathrm{G} 2$ for the different times of its monitoring, i.e. years 2004.25, 2006.25, 2008.25, 2010.25, 2011.25, 2012.25, 2013.25.

of our assumptions. The important result is that these quantities strongly depend on $\phi / D^{2}$, spanning orders of magnitude for $d_{\mathrm{BH}}$ corresponding to the observations (grey vertical bands). To this end, instead of the derived $R_{\text {in }}$, we adopted $r_{\mathrm{em}}$ as a free parameter in the hydrodynamic simulations in Section 3.

\subsubsection{Caveats}

The presented calculation contains a large number of approximations.

First of all, we neglect the role of shielding due to the 
dense shocked material around the free-wind region; this can lead to substantially lower ionization in the free-wind region.

Another extreme simplification is related to the assumed spherical symmetry of the calculation. Our Eq. 5 is based on the idea that G2 is hit at $R_{\text {out }}$ by $\phi / D^{2}$ photons coming from all directions, which is, of course, not the case. In reality, any surrounding young star will contribute to the illumination of G2 in a different way, dependent on its spectral class and position, hence making the flux not isotropically distributed on G2's surface. In addition to this, the pressure contributions (particularly the ram and tidal ones) shaping the free-wind region will make the free-wind surface asymmetric (for a discussion of the physics of stellar winds in the Galactic Centre, see Sec. 3 and Ballone et al. 2013, 2016; Christie et al. 2016).

Another caveat is related to the assumptions that ionizations and recombinations both occur istantaneously and that all the photons impinging on $R_{\text {out }}$ are totally absorbed by the free-wind region. However, as shown in Mapelli \& Ripamonti (2015), the timescales for these two processes might be very different. In our case, the recombination timescale is

$t_{\mathrm{rec}}(r)=\frac{1}{\alpha_{\mathrm{rec}} n_{\mathrm{i}}(r)} \approx 5 \times 10^{5} \frac{r_{14}^{2} v_{\mathrm{w}, 50}}{\dot{M}_{\mathrm{w},-7}} \mathrm{~s}$,

where $r_{14}$ is $r$ in units of $10^{14} \mathrm{~cm}$. The ionization timescale is

$t_{\mathrm{ion}}=\frac{4 \pi D^{2}}{\sigma_{\mathrm{H}} \phi} \approx 2 \times 10^{5} \frac{D_{\mathrm{pc}}^{2}}{\phi_{50}} \mathrm{~s}$

where $\sigma_{\mathrm{H}} \simeq 6.3 \times 10^{-18} \mathrm{~cm}^{2}$ is the cross section for neutral hydrogen and photons with energy $13.6 \mathrm{eV}$. An equilibrium between ionizations and recombinations can be assumed if $t_{\text {rec }}=t_{\text {ion }}$, which does not always hold for our assumptions. We also ignore that a certain number of photons (i.e., those passing through the outer envelope tangentially) might escape the free-wind region before ionizing any atom.

Finally, other physical processes could be important as well, such as collisional ionization from the wind (as already shown by Scoville \& Burkert 2013) or absorption of Lyc photons by the dust embedded in G2.

As already stated, the number of caveats listed here does not allow a strict use of the calculation for the modeling of G2's emission. Nonetheless, it powerfully shows that the contribution of the inner part of the outflow to the $\operatorname{Br} \gamma$ luminosity of G2 is not trivial.

\subsection{Resolution and numerical issues}

The constraining power of the absolute value of the $\mathrm{Br} \gamma$ luminosity has to be reconsidered, after the systematic study of the present 3D simulations.

First of all, the shocked material has a very filamentary nature; hence, if the filaments are not properly resolved, the density of the shocked material is reduced significantly.

Furthermore, as shown in Fig. 9 and 10, the shocked material is efficiently mixing with the atmosphere, moving to higher temperatures. In particular, as visible in Fig. 9, at early stages (e.g., in year 1950.25) the material closest
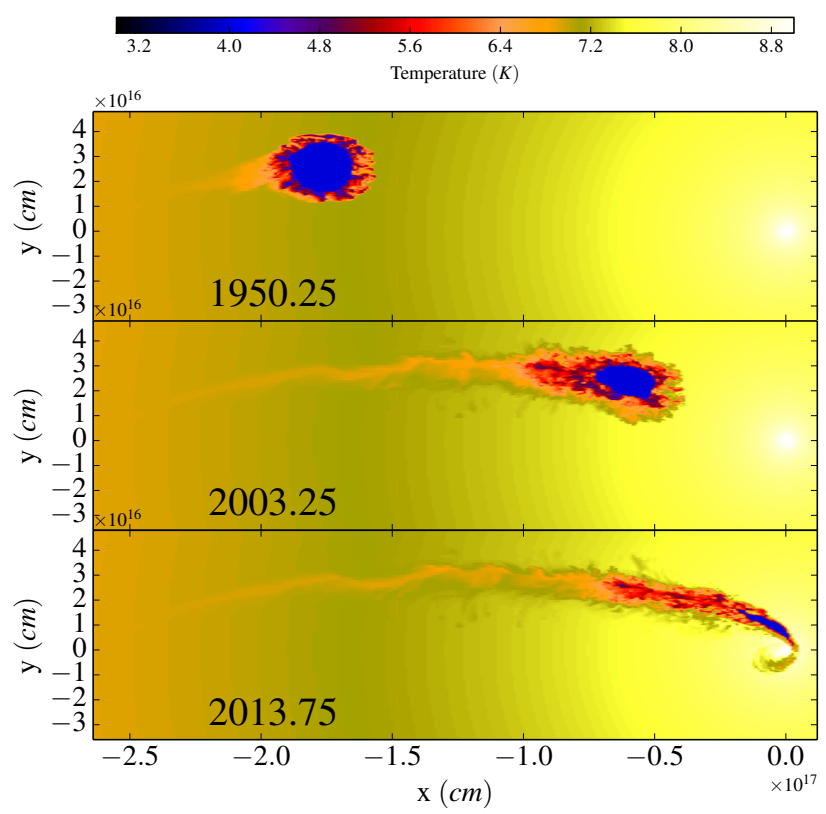

Figure 9. Temperature maps for the standard model. The domain plotted is a slice at $\mathrm{z}=0$.

to the free-wind region is at temperatures of around $10^{4} \mathrm{~K}$, i.e. the temperature of the injected material. However, the mixing becomes faster and faster as the source reaches its pericentre: at 2013.75, most of the shocked material is immediately increasing its temperature and a relatively small fraction is at temperatures below $10^{5} \mathrm{~K}$. The evolution of the phase plots for the shocked material in Fig. 10 might be misleading, since mixing with lighter material should also reduce its density with time. However, the diagram shows that the most luminous material increases its density (i.e., it moves to the right of the plot) as the source approaches pericentre; this is simply due to the fact that the outflow moves faster and it encounters higher density/pressure material on its way to the black hole. So, it is compressed more and reaching higher densities, as it gets closer to $\operatorname{Sgr} A^{*}$ (see also Fig. 1). On the other hand, the inner atmosphere is also hotter, hence the mixing leads to a large spread of the shocked material in the density-temperature phase space.

The mixing in our simulations is resolution dependent, since its nature is partly numerical. This has already been shown in Schartmann et al. (2015) for the diffuse cloud scenario. In the case of the present outflow model, mixing and resolution limitations are significant all along the orbital evolution, given the highly filamentary nature of the emitting material, and the effect of poor resolution is even less predictable.

The upper panel of Fig. 11 shows histograms for the luminosity of our standard model as a function of the density of the emitting material, for 2003.25 (i.e., the central panel of Fig. 10 collapsed along the temperature axis). The same histogram is plotted also for the simulation stLOWRES (the same as our standard model, but with half of the resolu- 


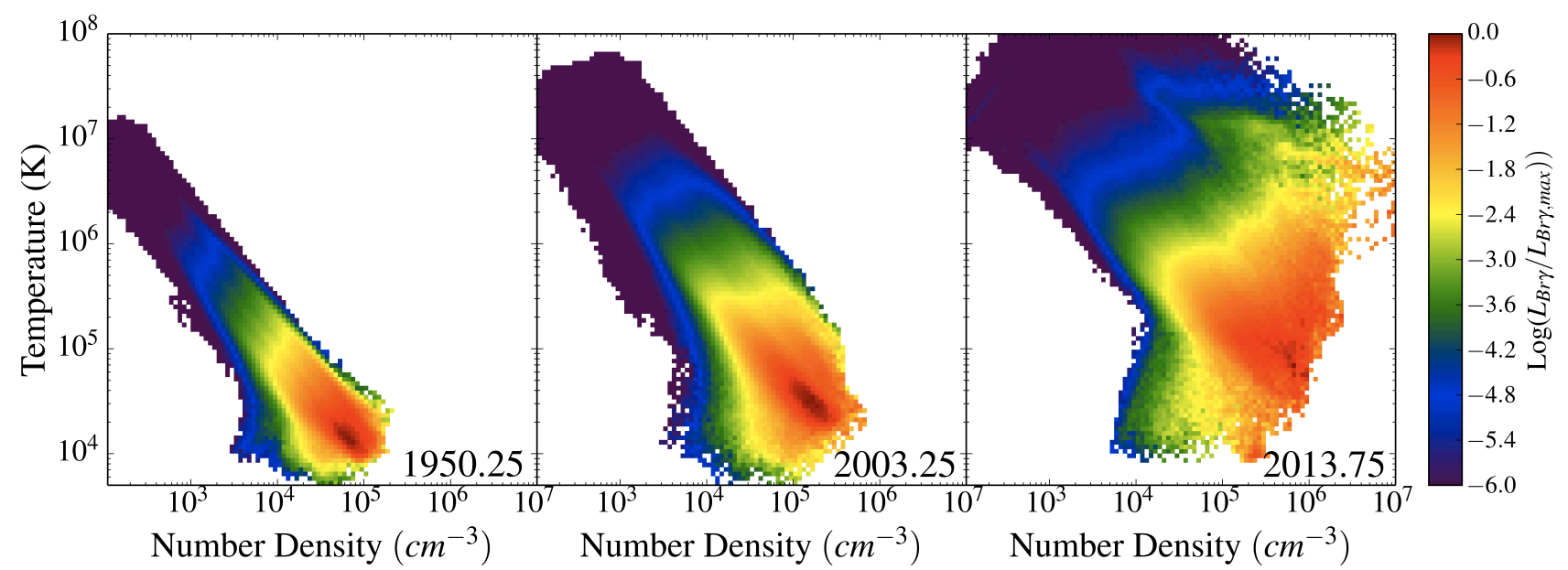

Figure 10. Phase plots for our standard model. Only the shocked material is included.
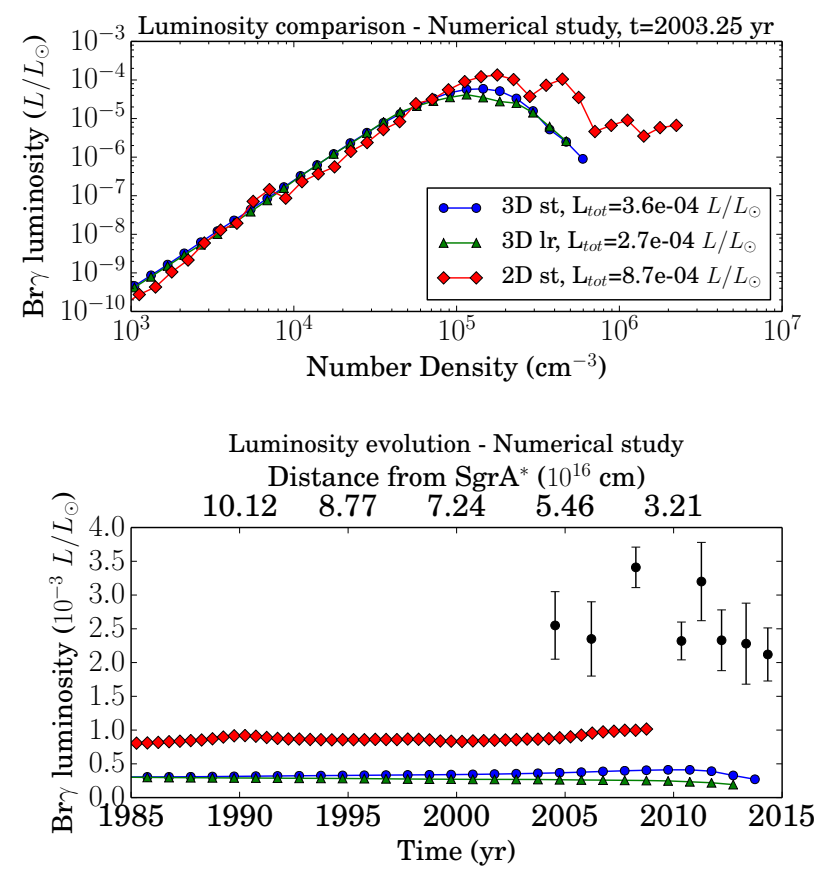

Figure 11. Luminosity comparison for our numerical study. Upper panel: contribution to the total luminosity by different density bins, in year 2003.25, for the standard model (blue circles), stLOWRES (green triangles) and st2D (red diamonds). Lower panel: luminosity evolution, close to pericentre, for the standard model, stLOWRES and st2D. Colours and symbols are the same as in the upper panel. The black points with error bars show the luminosities observed by Pfuhl et al. (2015).

tion) and for the simulation st2D (the same as the standard model, but in a $2 \mathrm{D}$ cylindrical fixed grid; see Table 1$)$. The luminosity distribution peaks ${ }^{1}$ around densities of roughly $10^{-19} \mathrm{~g} \mathrm{~cm}^{-3}$ for the outflow parameters of these three simulations. Though small, some discrepancy occurs between the two 3D simulations at different resolutions, particularly close to the peak of the distribution. This can account for the difference in the luminosity evolution, close to pericentre (see the lower panel of Fig. 11), between our standard model and model stLOWRES. The effect of resolution on the luminosity evolution is similar to the one visible in the resolution study of Schartmann et al. (2015).

A way more significant difference occurs, instead, between the luminosity curves of our standard model and its two-dimensional counterpart st2D. As already discussed in Ballone et al. (2013), simulations in 2D cylindrical coordinates suffer from some intrinsic numerical issues: in particular, the accumulation of material towards $R=0$, due to the tidal field of the SMBH, is artificially enhanced by the cylindrical symmetry and by the necessary reflective boundary conditions close to the axis of symmetry. For this reason, in order to compare with our standard model, we removed all the material at $R<1.25 \times 10^{14} \mathrm{~cm}$. However, still a significant contribution to the luminosity of st2D comes from densities higher than $2 \times 10^{-19} \mathrm{~g} \mathrm{~cm}^{-3}$, while this is not the case for the 3D standard model, showing that the artificial compression towards the axis of symmetry might have an effect on even larger distances from the axis. Furthermore, given the cylindrical symmetry, in the calculation of the luminosity the volume of every cell is obtained by a rotation of the cell around the $z$ axis (i.e., every cell has to be thought as a $3 \mathrm{D}$ annulus). As a consequence, the dense RayleighTaylor fingers forming immediately around the free-wind region might have a larger volume filling factor, compared to

1 The luminosity is given by the product between the density and the volume occupied by gas at that density. The latter is a decreasing function of the density, explaining the presence of a peak in the histogram. 
their 3D more realistic counterparts. All in all, there is a factor $\approx 2.5$ difference between the standard model and st2D, which forces us to also reconsider the luminosity curves obtained in the preliminary study of Ballone et al. (2013).

\subsection{Comparison with previous works}

In addition to the adopted dimensionality and coordinate system of the simulations, there are few additional differences between the simulations in Ballone et al. (2013) and the present ones. The first one is that the orbit has been updated from the one derived by Gillessen et al. (2013a) to the most-recent one derived by Gillessen et al. (2013b). The most up-to-date orbit has an orbital time and an apocentre distance that are roughly a factor of two larger than the one of the previous $2 \mathrm{D}$ simulations. This had the unfortunate effect of increasing the computational domain and double the integration time of our simulations, making the new simulations even more computationally expensive than previously expected.

Further, compared to Ballone et al. (2013), the massloss rate of our standard model has increased by roughly a factor 5 . This has been induced by the need of matching the PV diagrams shown in Fig. 2. In fact, the parameters of the best model in Ballone et al. (2013) are roughly corresponding to the LMDOT3D model described in Sec. 3.3, which is not able to match the size of G2 in the observed PV diagrams (see Fig. 6). The increase in the mass-loss rate of the best model is probably due to the more accurate comparison performed here, as well as to intrinsic differences between $2 \mathrm{D}$ and $3 \mathrm{D}$ simulations. Additionally, there are major differences in the absolute value of the luminosity, as discussed in Sec. 4.2 , due to differences in the resolution and perhaps in intrinsic differences between $3 \mathrm{D}$ cartesian and 2D cylindrical coordinates.

The choice of starting the simulations at apocentre makes the present results also very different from the ones in De Colle et al. (2014). In fact, the $\approx 200$ yr evolution of our models (compared to the 3 and $20 \mathrm{yr}$ chosen by De Colle et al. 2014) leads to a much more extended distribution of gas, as a result of the prolongated stripping of the RTI filaments of shocked wind. This larger filling volume is fundamental for matching the observed PV diagrams. However, no major instability forms in the simulations of De Colle et al. (2014), probably as a result of the too short evolution time of their models.

Major differences between our simulations and the ones in De Colle et al. (2014) also arise around pericentre, where the bow-shocks in their simulations - particularly those starting 3 years before pericentre - are becoming broader and underdense after the pericentre passage. This might be a consequence of their more sophisticated treatment of radiative cooling. The difference might also arise from the fact that, for those simulations, De Colle et al. (2014) did not artificially stabilize their atmosphere. This is allowing to compute the bow shock dynamics more properly, but it has the side effect of allowing the atmosphere to become convectively unstable (as clearly visible in Fig. 1 of De Colle et al. 2014).

Our work is also complementary to that by Zajaček et al. (2016). In this work, the evolution of the stellar wind shock is studied by means of the analytic solution of Wilkin
(1996). Such estimates have the advantage of having a simple but "linear" description of the interaction between the wind and the surrounding atmosphere; however, they lack more complex hydrodynamic processes that already arise from our simulations, even with our relatively simple physical treatment.

We must also point out that, besides lacking the detailed procedure to mock the instrumental effect on the processing of the simulation, the mock $\mathrm{Br} \gamma$ maps and the PV diagrams shown in Ballone et al. (2013), Gillessen et al. (2013b) and De Colle et al. (2014) include all the outflow material present in the simulations. This choice is arbitrary, since it depends on how much of the free-wind region is actually resolved in the simulation, and can produce PV diagrams with $\mathrm{Br} \gamma$ fluxes that are spanning several order of magnitudes, in evident inconsistency with the observations (compare to the upper panel of Fig. 2). Furthermore, as discussed in Sec. 4.1 and further on, the $\operatorname{Br} \gamma$ luminosity of a $1 / r^{2}$ density distribution depends on how much of it is actually ionized. Our more detailed post-processing of the simulation clearly shows that a more careful interpretation of the results must be applied, when dealing with this scenario.

Finally, this (and the previously mentioned) works focused on reproducing only G2, while the study presented in Ballone et al. (2016) tries to use the same model to simultaneously explain the presence of G2 and the following G2t. In this regard, even considering the weak constraining power of the $\mathrm{Br} \gamma$ luminosity, the present study shows that there should be a significant effect of the outflow parameters on the total luminosity of the shocked gas. Hence, the present standard model and the one in Ballone et al. (2016) are mutually exclusive. The model described in Ballone et al. (2016) has the advantage of being able to give G2 and G2t a common origin (even though the physical link between these two components is yet to be fully proven), but it has to be regarded as a proof of concept study and fine tuning of the model parameters is necessary to meet all observational constraints. The present standard model, on the other hand, is only able to reproduce G2, but it seems to have a $\mathrm{Br} \gamma$ luminosity that is closer to the observed one.

\subsection{On the nature of the source}

As already shown in Gillessen et al. (2012), the spectral properties of G2 exclude its association with a massive star, such as the S-stars. At the same time, the mass loss rates of our models are all too high for typical winds of low-mass stars in their main sequence phase.

Low-mass stars in their asymptotic giant branch or red giant phases might have comparable high mass loss rates (see, e.g., Whitelock et al. 2016). Stars in these phases have a giant envelope, that usually leads to outflow velocities of the order of their escape velocities, i.e., few tens of $\mathrm{km} / \mathrm{s}$. This is indeed the case for the standard model. However, those stars would appear too bright in $K_{s}$ band, compared to G2, and this possibility can be excluded.

The most appealing possibility is that the source is instead a young star, such as a $\mathrm{T}$ Tauri (see also Scoville \& Burkert 2013; Ballone et al. 2013). These objects are also producing winds, but they have much lower luminosities in $K_{s}$ band (see discussion in Scoville \& Burkert 2013). However, the parameters of the present 3D stan- 
dard model $\left(\dot{M}_{\mathrm{w}}=5 \times 10^{-7} \mathrm{M}_{\odot} \mathrm{yr}^{-1}\right.$ and $\left.v_{\mathrm{w}}=50 \mathrm{~km} \mathrm{~s}^{-1}\right)$ are somehow at the extreme end of the observed ranges for $\mathrm{T}$ Tauri's winds, which are $\dot{M}_{\mathrm{w}}=\left[10^{-12}, 10^{-7}\right] \mathrm{M}_{\odot} \mathrm{yr}^{-1}$ and $v_{\mathrm{w}}=[50,300] \mathrm{km} / \mathrm{s}$ from the observations (White \& Hillenbrand 2004). Given the short evolution time of our models $(\approx 200 \mathrm{yr}$ ), the standard model parameters could still correspond to a phase of exceptionally higher mass-loss. Indeed, there is a well established correlation between mass accretion and outflow rates for $\mathrm{T}$ Tauri objects, possibly being the consequence of outflows launched from the proto-stellar accretion disk (e.g., White \& Hillenbrand 2004; Edwards et al. 2006). In such a crowded environment and given the high tidal field of the black hole, the accretion (and outflow) rates might be enhanced compared to the typical star forming regions. Extremely massive outflows have been discovered, as e.g. for the case of DG Tau (Günther et al. 2009; White et al. 2014).

This problem can also be partially "cured" by assuming that the outflow is biconical, i.e., it is not occupying the full solid angle. As widely shown in literature, this is indeed a much more realistic assumption for the outflows from this kind of young stellar objects (e.g., Torbett 1984). In this case, Eq. 8 becomes

$R_{\text {out }, \text { conical }}=\left[\frac{\dot{M}_{\mathrm{w}} v_{\mathrm{w}}}{4 \pi\left(1-\cos \theta_{\text {open }}\right) P_{\mathrm{amb}}}\right]^{1 / 2}$,

where $\theta_{\text {open }}$ is the half opening angle of the outflow. So, for the same value of $R_{\text {out }}$, in the case of a biconical outflow, $\dot{M}_{\mathrm{w}}$ can be a factor $\left(1-\cos \theta_{\text {open }}\right)$ (i.e., up to a factor $\approx 10^{-2}$ for half opening angles as small as $\approx 10^{\circ}$ ) smaller compared to the isotropic case tested here. As shown in Sec. 3.3, the stagnation radius is on a 0 th order responsible for the size of the outflow; hence, to get sizes similar to the observed ones, lower mass-loss rates could be needed, compared to the ones found in our current simulations. However, the orientation of the biconical outflow with respect to the orbit is also likely effecting the distribution of the emitting material. This would add a further parameter to the present scenario and additional dedicated simulations would be needed to clarify this issue.

\subsection{Advantages and disadvantages of a compact source scenario}

As pointed out by the present and previous studies (Ballone et al. 2013; De Colle et al. 2014; Zajaček et al. 2016; Ballone et al. 2016), the compact source scenario is a highly parametric model, which makes the results strongly dependent on the assumptions made. Its intrinsic properties also make its study numerically challenging. Occam's razor would then suggest us that a diffuse cloud scenario (possibly without any hydrodynamical interaction with the accretion flow, as the one originally proposed by Gillessen et al. 2012) is to be preferred. However, more parameters can always offer more possibilities to reconcile the model and the observations.

For example, Pfuhl et al. (2015) showed that the total mass of the dust embedded in G2 is probably too low to make this component dynamically important. On the other hand, Witzel et al. (2014) showed that the dust stays compact even close to pericentre, compared to its gaseous counterpart. It is not clear why this should happen in a diffuse cloud scenario.
An outflow nature for G2 has the advantage of explaining both the extended (in position and velocity) nature of the gas component and the compactness of the dusty emission, if the latter is associated to a central young stellar object.

Another open question is related to the high eccentricity of G2's orbit. This could be well explained by a formation of G2 in colliding winds in the disk, if G2 is a clump of diffuse gas (Burkert et al. 2012; Schartmann et al. 2015; Calderón et al. 2016). Compared to stars, gas can more easily lose angular momentum (and energy) and the collision of stellar winds represents a very effective process, in this sense. However, the inner parsec is also very crowded with young stars (as young as T Tauri, see Sec. 4.4) and the S-stars can have similar orbital semi-major axes and can reach similarly high eccentricities.

A connection to a star could then be possible. The binary merger model of Witzel et al. (2014) could explain the dust properties and the high eccentricity of G2 (as later shown by Prodan et al. 2015; Stephan et al. 2016), but so far completely neglected the existence of a significant gaseous component associated with it. Outflow models are often invoked to explain the latter (see Sec. 1), but often they rely on - sometimes, too simple - analytical estimates. Despite the many limitations discussed in this section, our study represents the most complete attempt to include the several (often non-linear) processes involved in a compact source scenario and to compare to the observed properties of G2, e.g., by means of accurate mock PV diagrams.

Concerning the connection of G2 to G2t and G1 (see Sec. 1), Guillochon et al. (2014b) showed that these objects might result from the stripping of the outer envelope of a giant star by the tidal field of SgrA*. Hydrodynamical simulations of tidal disruptions of stars by SMBHs indeed show that these events might lead to the formation of a bound debris, streaming towards the SMBH on highly eccentric orbits (see also Guillochon et al. 2014a). The fragmentation of such a streamer might have led to G1, G2 and G2t. The formation of multiple clumps in colliding winds (Burkert et al. 2012; Schartmann et al. 2015; Calderón et al. 2016) is also a very reasonable explanation. G1 and the G2+G2t complex have very similar orbital and emission properties. This naturally suggests a common or similar origin. Proving that they were all born at the same location is less straight-forward; for example, G2's pre-pericentre and G1's post-pericentre orbits do not coincide perfectly and have a very different apocentre position. To reconcile the two, some loss of energy and angular momentum could have occured - mainly at pericentre -, due to the interaction of these clumps and the surrounding atmosphere (Pfuhl et al. 2015; McCourt et al. 2015; McCourt \& Madigan 2016; Madigan et al. 2017). However, Plewa et al. (2017) showed that G2 is keeping its original orbit even after pericentre, excluding the latter hypothesis of a strong drag of the atmosphere on these clumps. G2 and G1 could still be related, but the new findings show that these objects did not have exactly the same orbit, before pericentre. As shown by the HV3D model presented here and by the one in Ballone et al. (2016), an outflow with low enough density can efficiently form a tail of stripped gas (with properties similar to the observed G2t), although the source keeps on moving (and losing new material) on a purely Keplerian orbit.

The state-of-the-art models on G2's nature are all able to reproduce some of G2's properties, but also show lim- 
itations or are unable to explain other observables. Additionally, the pericentre evolution of G2 in simulations for the diffuse cloud scenario (Schartmann et al. 2012; Anninos et al. 2012; Schartmann et al. 2015) and in our simulations look very similar and the comparison to mock PV diagrams (Schartmann et al. 2015) shows that both models might be reconciled with observations. Hence, no final conclusion can be drawn, yet. The smoking gun for understanding whether a source is embedded in G2 could come in the next 5-10 years, when a decoupling between it and the previously outflowing gas might happen after pericentre, due to the increased cross section of the latter. At that point, the hydrodynamical interaction with the accretion flow would act on G2, but not on its central source and the newly emitted material, leading to the decoupling. The luminosity of the outflow material after pericentre can strongly depend on processes that cannot be too reliably captured by the present simulations, particularly during and right after the pericentre passage (see discussion is Sec. 4.2). The gas lost by the source before the pericentre passage in our simulations (and in those by Schartmann et al. 2012; Guillochon et al. 2014b; Schartmann et al. 2015) is decelerated by the hydrodynamical drag of the external accretion flow. At the same time, it is heating up, partially due to the mixing with the outer hot material, eventually leading to a substantial drop of its luminosity. Unfortunately, the mixing in the present simulations is mainly numerical. For this reason, no strong quantitative statement can currently be made, e.g., on the luminosity of old and new material and on the exact time of their decoupling. For our model we can, however, predict a non-symmetric behaviour of the gas, around the pericentre position, along its orbit (as opposed to what is expected for a purely ballistic diffuse cloud), and a "rebirth" of G2.

\section{SUMMARY}

In this work we presented 3D AMR simulations for a "compact source" scenario for G2, for which its gas component is produced by an outflow from a central source. Such a study is a natural follow-up of the study by Ballone et al. (2013), performed by means of $2 \mathrm{D}$ higher resolution simulations.

We can draw the following strong conclusions:

(i) Relatively massive $\left(\dot{M}_{\mathrm{w}}=5 \times 10^{-7} M_{\odot} \mathrm{yr}^{-1}\right)$ and slow (50 $\mathrm{km} \mathrm{s}^{-1}$ ), compared to main-sequence stars, outflows are needed to reproduce the emission properties of G2; furthermore, the central source must be a low mass star, due to observational constraints. This suggests that a possible source for G2 is a young stellar object, possibly a T Tauri star.

(ii) The appearance of such an outflow in the PV diagrams is strongly dependent on how much of its unperturbed region is actually emitting; if the material at distances smaller than roughly $100 \mathrm{AU}$ from the source dominates the emission, G2 would always look too compact both in size and in velocity - compared to the observations.

(iii) A reasonable comparison to the current SINFONI observations can be obtained both by the diffuse cloud simulations in Schartmann et al. (2015) and by the present ones. However, we might be able to understand whether G2 is generated by a source or if it is a simple gas-dust diffuse cloud in the next 5-10 years. For the case of a compact source, we should then be able to observe a decoupling between the dust and gas components and a new and "fresh" G2 should reform around the dusty one, later on.

Studying the "compact source" model presents more complications, compared to the "diffuse cloud" one. Still, the present can reproduce the $\operatorname{Br} \gamma$ observations and it has the advantage of being able to explain the simultaneous compactness of G2's dust component and extendedness of its gaseous one.

\section{ACKNOWLEDGEMENTS}

This project was supported by the Deutsche Forschungsgemeinschaft (DFG) priority program 1573 "Physics of the Interstellar Medium" and the DFG Cluster of Excellence "Origin and Structure of the Universe". Computer resources for this project have been provided by the Leibniz Supercomputing Center under grants: h0075, pr86re. Alessandro Ballone would like to thank Michela Mapelli, Andrea Gatto, James Guillochon, Jorge Cuadra, Diego Calderón and his PGN colleagues for useful discussions. Most of the simulation post-processing was carried out with the yt toolkit (Turk et al. 2011)

\section{REFERENCES}

Abarca D., Sądowski A., Sironi L., 2014, MNRAS, 440, 1125 Anninos P., Fragile P. C., Wilson J., Murray S. D., 2012, ApJ, 759, 132

Ballone A., et al., 2013, ApJ, 776, 13

Ballone A., et al., 2016, ApJ, 819, L28

Bartko H., et al., 2009, ApJ, 697, 1741

Borkar A., et al., 2016, MNRAS, 458, 2336

Bower G. C., et al., 2015, ApJ, 802, 69

Burkert A., Schartmann M., Alig C., Gillessen S., Genzel R., Fritz T. K., Eisenhauer F., 2012, ApJ, 750, 58

Calderón D., Ballone A., Cuadra J., Schartmann M., Burkert A., Gillessen S., 2016, MNRAS, 455, 4388

Chandler C. J., Sjouwerman L. O., 2014, The Astronomer's Telegram, 6247, 1

Christie I. M., Petropoulou M., Mimica P., Giannios D., 2016, MNRAS, 459, 2420

Clénet Y., et al., 2004a, A\&A, 417, L15

Clénet Y., et al., 2004b, A\&A, 424, L21

Clénet Y., Rouan D., Gratadour D., Marco O., Léna P., Ageorges N., Gendron E., 2005, A\&A, 439, L9

Crumley P., Kumar P., 2013, MNRAS, 436, 1955

De Colle F., Raga A. C., Contreras-Torres F. F., Toledo-Roy J. C., 2014, ApJ, 789, L33

Edwards S., Fischer W., Hillenbrand L., Kwan J., 2006, ApJ, 646, 319

Ferland G. J., 1980, PASP, 92, 596

Genzel R., Eisenhauer F., Gillessen S., 2010, Reviews of Modern Physics, 82, 3121

Ghez A. M., et al., 2005, ApJ, 635, 1087

Ghez A. M., et al., 2008, ApJ, 689, 1044

Gillessen S., Eisenhauer F., Trippe S., Alexander T., Genzel R., Martins F., Ott T., 2009, ApJ, 692, 1075

Gillessen S., et al., 2012, Nature, 481, 51

Gillessen S., et al., 2013a, ApJ, 763, 78

Gillessen S., et al., 2013b, ApJ, 774, 44

Guillochon J., Manukian H., Ramirez-Ruiz E., 2014a, ApJ, 783, 23 
Guillochon J., Loeb A., MacLeod M., Ramirez-Ruiz E., 2014b, ApJ, 786, L12

Günther H. M., Matt S. P., Li Z.-Y., 2009, A\&A, 493, 579

Haggard D., et al., 2014, The Astronomer's Telegram, 6242, 1

Hamann F., Ferland G., 1999, ARA\&A, 37, 487

Lu J. R., Ghez A. M., Hornstein S. D., Morris M. R., Becklin E. E., Matthews K., 2009, ApJ, 690, 1463

Madigan A.-M., McCourt M., O'Leary R. M., 2017, MNRAS, 465, 2310

Mapelli M., Ripamonti E., 2015, ApJ, 806, 197

Martins F., Genzel R., Hillier D. J., Eisenhauer F., Paumard T., Gillessen S., Ott T., Trippe S., 2007, A\&A, 468, 233

McCourt M., Madigan A.-M., 2016, MNRAS, 455, 2187

McCourt M., O'Leary R. M., Madigan A.-M., Quataert E., 2015, MNRAS, 449, 2

Meyer F., Meyer-Hofmeister E., 2012, A\&A, 546, L2

Mignone A., Bodo G., Massaglia S., Matsakos T., Tesileanu O., Zanni C., Ferrari A., 2007, ApJS, 170, 228

Mignone A., Zanni C., Tzeferacos P., van Straalen B., Colella P., Bodo G., 2012, ApJS, 198, 7

Miralda-Escudé J., 2012, ApJ, 756, 86

Mossoux E., et al., 2016, A\&A, 589, A116

Murray-Clay R. A., Loeb A., 2012, Nature Communications, 3

Narayan R., Özel F., Sironi L., 2012, ApJ, 757, L20

Osterbrock D. E., Ferland G. J., 2006, Astrophysics of gaseous nebulae and active galactic nuclei

Paumard T., et al., 2006, ApJ, 643, 1011

Pfuhl O., et al., 2015, ApJ, 798, 111

Phifer K., et al., 2013, ApJ, 773, L13

Plewa P. M., et al., 2017, ApJ, 840, 50

Ponti G., et al., 2015, MNRAS, 454, 1525

Prodan S., Antonini F., Perets H. B., 2015, ApJ, 799, 118

Sądowski A., Sironi L., Abarca D., Guo X., Özel F., Narayan R., 2013a, MNRAS, 432, 478

Sądowski A., Narayan R., Sironi L., Özel F., 2013b, MNRAS, 433, 2165

Schartmann M., Burkert A., Alig C., Gillessen S., Genzel R., Eisenhauer F., Fritz T. K., 2012, ApJ, 755, 155

Schartmann M., et al., 2015, ApJ, 811, 155

Scoville N., Burkert A., 2013, ApJ, 768, 108

Shcherbakov R. V., 2014, ApJ, 783, 31

Stephan A. P., Naoz S., Ghez A. M., Witzel G., Sitarski B. N., Do T., Kocsis B., 2016, MNRAS, 460, 3494

Torbett M. V., 1984, ApJ, 278, 318

Trani A. A., Mapelli M., Spera M., Bressan A., 2016, ApJ, 831, 61

Turk M. J., Smith B. D., Oishi J. S., Skory S., Skillman S. W., Abel T., Norman M. L., 2011, The Astrophysical Journal Supplement Series, 192, 9

Valencia-S. M., et al., 2015, ApJ, 800, 125

White R. J., Hillenbrand L. A., 2004, ApJ, 616, 998

White M. C., McGregor P. J., Bicknell G. V., Salmeron R., Beck T. L., 2014, MNRAS, 441, 1681

Whitelock P. A., Boyer M., Höfner S., Wittkowski M., Zijlstra A. A., 2016, in 19th Cambridge Workshop on Cool Stars, Stellar Systems, and the Sun (CS19). p. 5 (arXiv:1609.07954), doi:10.5281/zenodo.154075

Wilkin F. P., 1996, ApJ, 459, L31

Witzel G., et al., 2014, ApJ, 796, L8

Zajaček M., Karas V., Eckart A., 2014, A\&A, 565, A17

Zajaček M., Eckart A., Karas V., Kunneriath D., Shahzamanian B., Sabha N., Mužić K., Valencia-S. M., 2016, MNRAS, 455, 1257

Zajaček M., et al., 2017, preprint, (arXiv:1704.03699)

This paper has been typeset from a $\mathrm{T}_{\mathrm{E}} \mathrm{X} / \mathrm{LAT} \mathrm{T} \mathrm{X}$ file prepared by the author. 